\title{
Supporting Information for: \\ More Than a First Flush: Urban Creek Storm Hydrographs Demonstrate Broad Contaminant Pollutographs
}

Katherine T. Peter ${ }^{1,2 *}$, Fan Hou, ${ }^{1,3}$ Zhenyu Tian ${ }^{1,2}$, Christopher $\mathrm{Wu}^{2}$, Matt Goehring, ${ }^{4}$ Fengmao Liu, ${ }^{3}$ Edward P. Kolodziej ${ }^{1,2,5}$

${ }^{1}$ Center for Urban Waters, Tacoma WA, 98421 USA

${ }^{2}$ Interdisciplinary Arts and Sciences, University of Washington Tacoma, Tacoma, WA, 98421 USA

${ }^{3}$ Department of Applied Chemistry, College of Science, China Agricultural University, Beijing, 100193 China

${ }^{4}$ Green/Duwamish \& Central Puget Sound Watershed (WRIA 9), King County, Seattle, WA 98104 USA

${ }^{5}$ Department of Civil and Environmental Engineering, University of Washington, Seattle, WA, 98195 USA

April 8, 2020

30 pages, 8 tables, and 10 figures

Revised for submission to:

Environmental Science \& Technology

*Corresponding author contact information

Email: katherine.peter@ nist.gov 
Table S1. Chemical standards and suppliers.

Table S2. Isotope labeled internal standards.

Table S3. LC-MS/MS $\log K_{o w}$ values, minimum quantification limits (MQLs) and minimum detection limits (MDLs).

Table S4. Profinder and Mass Profiler Professional settings.

Table S5. Internal standard peak area ratios.

Table S6. Concentrations of LC-MS/MS targeted analytes.

Table S7. DPG, HMMM, and DCU concentrations in TWP leachate and loading in TWP.

Table S8. Peak area and detection frequency of identified HRMS compounds (in separate Excel file).

Figure S1. Map of Miller Creek Watershed.

Figure S2. Photos of Miller Creek.

Figure S3. Median internal standard peak area ratios.

Figure S4. Storm hydrograph characteristics in Miller Creek.

Figure S5. Relative water quality composition by contaminant class.

Figure S6. Concentrations of LC-MS/MS targeted analytes

Figure S7. Surrogate metrics of water quality dynamics specific to coho salmon URMS

Figure S8. Peak area of identified HRMS compounds.

Figure S9. Distribution of HRMS peak area, with compound detection categorized by flow condition.

Figure S10. Distribution of HRMS compound maximum abundance, categorized by polarity.

Chemicals. Acetic acid (>99.7\%) and ammonium acetate (HPLC grade, 97.8\%) were purchased from VWR Scientific (Radnor, PA, USA). Methanol (MeOH: OPTIMA® grade) and Triton X100 (octylphenol ethoxylate, scintillation grade; Research Products International) were obtained from Fisher Scientific (Fair Lawn, NJ, USA). A water purification system (Thermo Barnstead Nanopure Diamond UV, Dubuque, IA, USA) provided $18 \mathrm{M} \Omega$ water. Chemical standards and suppliers are provided in Tables S1-S2.

Raw data files (including MS/MS spectra) are available upon request.

Table S1. Chemical standards with CAS number, molecular formula, and supplier.

\begin{tabular}{|c|c|c|c|}
\hline Compound & CAS & $\begin{array}{l}\text { Molecular } \\
\text { formula }\end{array}$ & Supplier \\
\hline Hexa(methoxymethyl)melamine & $3089-11-0$ & C15H30N6O6 & Combi-Blocks ltd \\
\hline $\begin{array}{l}\text { N-cyclohexyl-1,3-benzothiazol-2- } \\
\text { amine }\end{array}$ & $28291-75-0$ & C13H16N2S & Enamine \\
\hline 1,3-Diphenylguanidine & $102-06-7$ & C13H13N3 & \multirow{7}{*}{ Sigma-Aldrich } \\
\hline Benzotriazole & $95-14-7$ & C6H5N3 & \\
\hline 5-methyl-1H-Benzotriazole & $136-85-6$ & C7H7N3 & \\
\hline 2-amino-Benzothiazole & $136-95-8$ & C7H6N2S & \\
\hline 2-hydroxy-Benzothiazole & $934-34-9$ & C7H5NOS & \\
\hline 2-(4-morpholinyl)benzothiazole & $4225-26-7$ & C11H12N2OS & \\
\hline Clothanidin & $210880-92-5$ & C6N5H8SO2Cl & \\
\hline
\end{tabular}




\begin{tabular}{|c|c|c|c|}
\hline Imidacloprid & $138261-41-3$ & C9H10ClN5O2 & \\
\hline Thiamethoxam & $153719-23-4$ & C8H10CIN5O3S & \\
\hline Carbendazim & $10605-21-7$ & C9H9N3O2 & \\
\hline Iprodione & $36734-19-7$ & $\mathrm{C} 13 \mathrm{H} 13 \mathrm{Cl} 2 \mathrm{~N} 3 \mathrm{O} 3$ & \\
\hline Pentachlorophenol (PCP) & $87-86-5$ & $\mathrm{C} 6 \mathrm{HCl} 5 \mathrm{O}$ & \\
\hline Diuron & $330-54-1$ & $\mathrm{C} 9 \mathrm{H} 10 \mathrm{Cl} 2 \mathrm{~N} 2 \mathrm{O}$ & \\
\hline Mecoprop & $7085-19-0$ & $\mathrm{C} 10 \mathrm{H} 11 \mathrm{ClO} 3$ & \\
\hline Prometon & $1610-18-0$ & C10H19N5O & \\
\hline Caffeine & $58-08-2$ & C8H10N4O2 & \\
\hline Cetirizine & $83881-52-1$ & $\mathrm{C} 21 \mathrm{H} 27 \mathrm{Cl} 3 \mathrm{~N} 2 \mathrm{O} 3$ & \\
\hline Cotinine & $486-56-6$ & $\mathrm{C} 10 \mathrm{H} 12 \mathrm{~N} 2 \mathrm{O}$ & \\
\hline DEET & $134-62-3$ & $\mathrm{C} 12 \mathrm{H} 17 \mathrm{NO}$ & \\
\hline Diclofenac & $15307-86-5$ & $\mathrm{C} 14 \mathrm{H} 10 \mathrm{Cl} 2 \mathrm{NNaO} 2$ & \\
\hline Ibuprofen & $15687-27-1$ & $\mathrm{C} 13 \mathrm{H} 18 \mathrm{O} 2$ & \\
\hline 1,3-Dicyclohexylurea & $2387-23-7$ & $\mathrm{C} 13 \mathrm{H} 24 \mathrm{~N} 2 \mathrm{O}$ & \\
\hline Bisphenol A & $80-05-7$ & $\mathrm{C} 15 \mathrm{H} 16 \mathrm{O} 2$ & \\
\hline Caprolactam & $105-60-2$ & C6H11NO & \\
\hline 4-Nonylphenol & $84852-15-3$ & $\mathrm{C} 15 \mathrm{H} 24 \mathrm{O}$ & \\
\hline 4-tert-Octylphenol & $140-66-9$ & $\mathrm{C} 14 \mathrm{H} 22 \mathrm{O}$ & \\
\hline $\begin{array}{l}\text { poly(ethylene glycol) (PEG; average } \\
\left.M_{n} 400\right)\end{array}$ & $25322-68-3$ & $\mathrm{C}(2 \mathrm{n}-2) \mathrm{H}(4 \mathrm{n}-2) \mathrm{On}$ & \\
\hline PEG methyl ether (average $M_{n} 550$ ) & $9004-74-4$ & $\mathrm{C}(2 \mathrm{n}-1) \mathrm{H}(4 \mathrm{n}) \mathrm{On}$ & \\
\hline PEG dimethyl ether (average $\mathrm{M}_{\mathrm{n}} \sim 250$ ) & $24991-55-7$ & $\mathrm{C}(2 \mathrm{n}) \mathrm{H}(4 \mathrm{n}+2) \mathrm{On}$ & \\
\hline $\begin{array}{l}\text { poly(propylene glycol) (PPG; average } \\
M_{n} \sim 425\end{array}$ & $25322-69-4$ & $\mathrm{C}(3 \mathrm{n}-3) \mathrm{H}(6 \mathrm{n}-4) \mathrm{On}$ & \\
\hline Tris(2-butoxyethyl)phosphate & $78-51-3$ & C18H39O7P & \\
\hline 2,6-dichlorobenzamide (BAM) & $2008-58-4$ & $\mathrm{C} 7 \mathrm{H} 5 \mathrm{Cl} 2 \mathrm{NO}$ & \\
\hline Nicotine & $54-11-5$ & C10H14N2 & \\
\hline Methamphetamine & $537-46-2$ & $\mathrm{C} 10 \mathrm{H} 15 \mathrm{~N}$ & \\
\hline Denatonium & $47324-98-1$ & $\mathrm{C} 21 \mathrm{H} 29 \mathrm{~N} 2 \mathrm{O}+$ & \\
\hline 4-nitrosodiphenylamine & $156-10-5$ & $\mathrm{C} 12 \mathrm{H} 10 \mathrm{~N} 2 \mathrm{O}$ & \\
\hline 2-benzothiazolesulfonic acid & $941-57-1$ & C7H5NO3S2 & \\
\hline Fipronil & $120068-37-3$ & $\mathrm{C} 12 \mathrm{H} 4 \mathrm{Cl} 2 \mathrm{~F} 6 \mathrm{~N} 4 \mathrm{OS}$ & \multirow{5}{*}{$\begin{array}{l}\text { Toronto Research } \\
\text { Chemicals }\end{array}$} \\
\hline Diazinon & $333-41-5$ & $\mathrm{C} 12 \mathrm{H} 21 \mathrm{~N} 2 \mathrm{O} 3 \mathrm{PS}$ & \\
\hline 4-Nitrophenol & $100-02-7$ & C6H5NO3 & \\
\hline Metformin & $1115-70-4$ & C4H11N5 & \\
\hline Triclosan & $3380-34-5$ & $\mathrm{C} 12 \mathrm{H} 7 \mathrm{Cl} 3 \mathrm{O} 2$ & \\
\hline SDPA-C4C8 & $184378-08-3$ & $\mathrm{C} 24 \mathrm{H} 35 \mathrm{~N}$ & \multirow{5}{*}{$\begin{array}{l}\text { Aquatic Contaminants } \\
\text { Research Division } \\
\text { (Canada) }\end{array}$} \\
\hline SDPA-C8C8 & $184378-08-3$ & $\mathrm{C} 28 \mathrm{H} 43 \mathrm{~N}$ & \\
\hline SDPA-C9C9 & $68608-79-7$ & $\mathrm{C} 30 \mathrm{H} 47 \mathrm{~N}$ & \\
\hline SDPA-diAMS & 10081-67-1 & $\mathrm{C} 30 \mathrm{H} 31 \mathrm{~N}$ & \\
\hline BTZ \& UV-234 & 70321-86-7 & $\mathrm{C} 30 \mathrm{H} 29 \mathrm{~N} 3 \mathrm{O}$ & \\
\hline
\end{tabular}


Table S2. Isotope labeled internal standards, with CAS number, supplier, and working concentration.

\begin{tabular}{|l|l|l|c|}
\hline \multicolumn{1}{|c|}{$\begin{array}{c}\text { Isotope labeled internal } \\
\text { standard }\end{array}$} & \multicolumn{1}{|c|}{ CAS } & \multicolumn{1}{|c|}{ Supplier } & $\begin{array}{c}\text { Working concentration } \\
\text { in 1 L sample (ng/L) }\end{array}$ \\
\hline 4-nitrophenol-d4 & $93951-79-2$ & Sigma Aldrich & 50 \\
\hline Cotinine-d3 & $110952-70-0$ & Sigma Aldrich & 10 \\
\hline Caffeine-13C3 & $78072-66-9$ & Sigma Aldrich & 10 \\
\hline Imidacloprid-d4 & $1015855-75-0$ & Toronto Research Chemicals & 20 \\
\hline 5-methyl-1H-benzotriazole-d6 & $1246820-65-4$ & Toronto Research Chemicals & 20 \\
\hline Carbendazim-d4 & $291765-95-2$ & CDN Isotopes & 5 \\
\hline Diclofenac-d4 & $153466-65-0$ & Toronto Research Chemicals & 20 \\
\hline Ibuprofen-d3 & $121662-14-4$ & Sigma Aldrich & 200 \\
\hline 1,3-diphenyl-d10-urea & $108009-46-7$ & Toronto Research Chemicals & 5 \\
\hline DEET-d7 & $1219799-37-7$ & CDN Isotopes & 20 \\
\hline Bisphenol A-d8 & $92739-58-7$ & Toronto Research Chemicals & 100 \\
\hline Diuron-d6 & $1007536-67-5$ & Toronto Research Chemicals & 10 \\
\hline Cetirizine-d8 & $774596-22-4$ & Toronto Research Chemicals & 1 \\
\hline Prometon-d3 & $1219803-43-6$ & CDN Isotopes & 10 \\
\hline Diazinon-d10 & $100155-47-3$ & Toronto Research Chemicals & 25 \\
\hline Triclosan-d3 & $1020719-98-5$ & CDN Isotopes & 10 \\
\hline Bis(2-ethylhexyl)phthalate-d4 & $93951-87-2$ & CDN Isotopes & \\
\hline
\end{tabular}


Table S3. Polarity (as $\left.\log K_{o w}\right)^{1,2}$ and minimum quantification limits (MQLs) and minimum detection limits (MDLs) (in ng/L) for 35 LC-MS/MS quantified contaminants. Analytes marked with an asterisk (*) are semi-quantitative, as only one ion transition was monitored.

\begin{tabular}{|c|c|c|c|c|c|c|c|c|c|c|c|c|c|c|}
\hline \multirow{3}{*}{$\begin{array}{l}\text { Contaminant } \\
\text { Group }\end{array}$} & \multirow{3}{*}{$\begin{array}{c}\text { Sampling Date: } \\
\text { Event Type: } \\
\text { Compound }\end{array}$} & \multirow{2}{*}{\multicolumn{3}{|c|}{$\begin{array}{c}\text { July 26, } 2018 \\
\text { Baseflow }\end{array}$}} & \multirow{2}{*}{\multicolumn{2}{|c|}{$\begin{array}{c}\text { August 15, } 2018 \\
\text { Baseflow }\end{array}$}} & \multirow{2}{*}{\multicolumn{2}{|c|}{$\begin{array}{c}\text { September 18, } \\
2018 \\
\text { Baseflow }\end{array}$}} & \multirow{2}{*}{\multicolumn{2}{|c|}{$\begin{array}{c}\text { Oct } 25-27, \\
2018 \\
\text { Storm }\end{array}$}} & \multirow{2}{*}{\multicolumn{2}{|c|}{$\begin{array}{c}\text { Nov 1-3, } 2018 \\
\text { Storm }\end{array}$}} & \multirow{2}{*}{\multicolumn{2}{|c|}{$\begin{array}{c}\text { Dec 10-12, } \\
2018 \\
\text { Storm }\end{array}$}} \\
\hline & & & & & & & & & & & & & & \\
\hline & & $\log K_{o w}$ & MQL & MDL & MQL & MDL & MQL & MDL & MQL & MDL & MQL & MDL & MQL & MDL \\
\hline \multirow{3}{*}{$\begin{array}{l}\text { Vehicle- } \\
\text { derived }\end{array}$} & $\begin{array}{l}\text { 1,3- } \\
\text { Diphenylguanidine }\end{array}$ & 2.49 & 1.4 & 1.1 & 0.74 & 0.65 & 1.2 & 0.77 & 1.2 & 1.0 & 2.4 & 0.9 & 4.3 & 2.7 \\
\hline & HMMM & 2.14 & 0.21 & 0.06 & 0.21 & 0.06 & 0.21 & 0.06 & 0.21 & 0.06 & 0.21 & 0.06 & 0.21 & 0.06 \\
\hline & NCBA & 3.39 & 0.5 & 0.43 & 0.9 & 0.76 & 1.5 & 1.5 & 2.5 & 1.7 & 0.5 & 0.42 & 1.1 & 1.1 \\
\hline \multirow{5}{*}{$\begin{array}{c}\text { Benzothiazole } \\
/ \\
\text { Benzotriazole }\end{array}$} & Benzotriazole & 1.44 & 2.0 & 0.61 & 2.0 & 0.61 & 2.0 & 0.61 & 2.0 & 0.61 & 2.0 & 0.61 & 2.0 & 0.61 \\
\hline & 5-methylbenzotriazole & 1.52 & 0.14 & 0.04 & 0.14 & 0.04 & 0.14 & 0.04 & 0.14 & 0.04 & 0.14 & 0.04 & 0.14 & 0.04 \\
\hline & $\begin{array}{l}\text { 2-amino- } \\
\text { benzothiazole }\end{array}$ & 1.63 & 0.74 & 0.22 & 0.74 & 0.22 & 0.74 & 0.22 & 0.74 & 0.22 & 0.74 & 0.22 & 0.74 & 0.22 \\
\hline & $\begin{array}{l}\text { 2-hydroxy- } \\
\text { benzothiazole }\end{array}$ & 1.78 & 17 & 5.2 & 17 & 5.2 & 17 & 5.2 & 17 & 5.2 & 17 & 5.2 & 17 & 5.2 \\
\hline & $\begin{array}{l}\text { 2-(4-morpholinyl)- } \\
\text { benzothiazole }\end{array}$ & 2.09 & 0.90 & 0.27 & 0.90 & 0.27 & 0.90 & 0.27 & 0.90 & 0.27 & 0.90 & 0.27 & 0.90 & 0.27 \\
\hline \multirow{4}{*}{$\begin{array}{l}\text { Industrial } \\
\text { Chemicals }\end{array}$} & 1,3-Dicyclohexylurea & 3.16 & 1.3 & 1.0 & 1.5 & 1.0 & 16 & 4.8 & 1.3 & 0.6 & 1.4 & 0.7 & 12 & 6.4 \\
\hline & Bisphenol A & 3.29 & 2.5 & 0.75 & 2.5 & 0.75 & 2.5 & 0.75 & 2.5 & 0.75 & 2.5 & 0.75 & 2.5 & 0.75 \\
\hline & 4-Nonylphenol* & 6.03 & 40 & 12 & 40 & 0.36 & 40 & 0.36 & 40 & 0.36 & 40 & 0.36 & 40 & 0.36 \\
\hline & 4-tert-Octylphenol & 5.56 & 53 & 16 & 53 & 0.33 & 53 & 0.33 & 53.0 & 0.33 & 53 & 0.33 & 53 & 0.33 \\
\hline \multirow{10}{*}{ Pesticides } & Clothianidin & 0.7 & 0.29 & 0.09 & 0.29 & 0.09 & 0.29 & 0.09 & 0.29 & 0.09 & 0.29 & 0.09 & 0.29 & 0.09 \\
\hline & Imidacloprid & 0.57 & 0.16 & 0.05 & 0.16 & 0.05 & 0.16 & 0.05 & 0.16 & 0.05 & 0.16 & 0.05 & 0.16 & 0.05 \\
\hline & Thiamethoxam & -0.118 & 3.5 & 1.06 & 3.5 & 1.06 & 3.5 & 1.1 & 3.5 & 1.06 & 3.5 & 1.1 & 3.5 & 1.06 \\
\hline & Fipronil & 4.34 & 0.01 & $4 \mathrm{E}-03$ & 0.01 & $4 \mathrm{E}-03$ & 0.01 & 4E-03 & 0.01 & $4 \mathrm{E}-03$ & 0.01 & $4 \mathrm{E}-03$ & 0.01 & $4 \mathrm{E}-03$ \\
\hline & Carbendazim & 1.52 & 2.8 & 2.6 & 0.7 & 0.6 & 1.1 & 1.0 & 1.7 & 1.1 & 0.8 & 0.6 & 1.0 & 0.9 \\
\hline & Pentachlorophenol & 4.86 & 2.2 & 0.65 & 2.2 & 0.65 & 2.2 & 0.65 & 2.2 & 0.65 & 2.2 & 0.65 & 2.2 & 0.65 \\
\hline & Diazinon & 3.81 & 0.49 & 0.15 & 0.49 & 0.15 & 0.49 & 0.15 & 0.49 & 0.15 & 0.49 & 0.15 & 0.49 & 0.15 \\
\hline & Diuron & 2.68 & 0.17 & 0.05 & 0.17 & 0.05 & 0.17 & 0.05 & 0.17 & 0.05 & 0.17 & 0.05 & 0.17 & 0.05 \\
\hline & Mecoprop & 3.13 & 0.24 & 0.07 & 0.24 & 0.07 & 0.24 & 0.07 & 0.24 & 0.07 & 0.24 & 0.07 & 0.24 & 0.07 \\
\hline & Prometon & 2.99 & 0.4 & 0.38 & 0.2 & 0.24 & 0.01 & 0.00 & 0.01 & 0.00 & 0.01 & 0.00 & 0.01 & 0.00 \\
\hline
\end{tabular}




\begin{tabular}{|c|c|c|c|c|c|c|c|c|c|c|c|c|c|c|}
\hline \multirow{7}{*}{ PPCPs } & Caffeine & -0.07 & 1.1 & 0.34 & 1.1 & 0.34 & 1.1 & 0.34 & 1.1 & 0.34 & 1.1 & 0.34 & 1.1 & 0.34 \\
\hline & Cetirizine & 1.7 & 0.04 & 0.01 & 0.04 & 0.01 & 0.04 & 0.01 & 0.04 & 0.01 & 0.04 & 0.01 & 0.04 & 0.01 \\
\hline & Cotinine & 0.07 & 0.8 & 0.6 & 1.0 & 0.6 & 0.5 & 0.4 & 4.0 & 1.6 & 0.8 & 0.6 & 2.5 & 1.3 \\
\hline & DEET & 2.13 & 5.8 & 5.7 & 4.5 & 4.5 & 2.2 & 2.2 & 4.1 & 2.8 & 3.1 & 2.5 & 7.2 & 4.2 \\
\hline & Diclofenac & 3.91 & 2.9 & 0.87 & 2.9 & 0.87 & 2.9 & 0.87 & 2.9 & 0.87 & 2.9 & 0.87 & 2.9 & 0.87 \\
\hline & Ibuprofen* & 3.67 & 11 & 3.2 & 11 & 3.2 & 11 & 3.2 & 11 & 3.2 & 11 & 3.2 & 11 & 3.2 \\
\hline & Triclosan* & 4.79 & 1.2 & 0.37 & 1.2 & 0.37 & 1.2 & 0.37 & 1.2 & 0.37 & 1.2 & 0.37 & 1.2 & 0.37 \\
\hline \multirow{6}{*}{$\begin{array}{c}\text { SDPA/BZT- } \\
\text { UV }\end{array}$} & SDPA-diAMS & 8.5 & 0.12 & 0.04 & 0.12 & 0.04 & 0.12 & 0.04 & 0.12 & 0.04 & 0.12 & 0.04 & 0.12 & 0.04 \\
\hline & SDPA-C4C8 & 9.3 & 0.15 & 0.04 & 0.15 & 0.04 & 0.15 & 0.04 & 0.15 & 0.04 & 0.15 & 0.04 & 0.15 & 0.04 \\
\hline & SDPA-C8C8* & 11.2 & 0.38 & 0.11 & 0.38 & 0.11 & 0.38 & 0.11 & 0.38 & 0.11 & 0.38 & 0.11 & 0.38 & 0.11 \\
\hline & SDPA-C9C9* & 12.2 & 0.75 & 0.22 & 0.75 & 0.22 & 0.75 & 0.22 & 0.75 & 0.22 & 0.75 & 0.22 & 0.75 & 0.22 \\
\hline & BZT\&UV-234 & 7.7 & 0.09 & 0.03 & 0.09 & 0.03 & 0.09 & 0.03 & 0.09 & 0.03 & 0.09 & 0.03 & 0.09 & 0.03 \\
\hline & BZT\&UV-326 & 5.6 & 0.57 & 0.17 & 0.57 & 0.17 & 0.57 & 0.17 & 0.57 & 0.17 & 0.57 & 0.17 & 0.57 & 0.17 \\
\hline
\end{tabular}


Table S4. Profinder and Mass Profiler Professional (MPP) HRMS data extraction and alignment settings.

\begin{tabular}{|c|c|c|}
\hline $\begin{array}{l}\text { Profinder Batch } \\
\text { Recursive Feature } \\
\text { Extraction Wizard } \\
\text { Step }\end{array}$ & Category & Setting \\
\hline \multirow{3}{*}{$\begin{array}{c}\text { Molecular Feature } \\
\text { Extraction (MFE) - } \\
\text { Extraction Parameters }\end{array}$} & $\begin{array}{c}\text { Noise peak } \\
\text { height }\end{array}$ & $>=400$ counts \\
\hline & Ion Species & $\begin{array}{l}+\mathrm{H},+\mathrm{Na},+\mathrm{NH} 4 \text { (salt- } \\
\text { dominated positive ions) }\end{array}$ \\
\hline & Isotope model & Common organic molecules \\
\hline \multirow{2}{*}{$\begin{array}{c}\text { Compound Binning and } \\
\text { Alignment }\end{array}$} & RT window & $0.6 \mathrm{~min}$ \\
\hline & Mass window & $30 \mathrm{ppm}$ \\
\hline \multirow{3}{*}{$\begin{array}{c}\text { MFE - Post-Processing } \\
\text { Filters }\end{array}$} & Absolute height & $>=5000$ counts \\
\hline & Score (MFE) & $>=70$ \\
\hline & $\begin{array}{l}\text { Satisfy MFE } \\
\text { conditions in }\end{array}$ & $>=2$ files \\
\hline \multirow[t]{2}{*}{$\begin{array}{l}\text { Find by Ion-Matching } \\
\text { Tolerances and Scoring }\end{array}$} & Scoring & $\begin{array}{l}\text { Mass score (100), isotope } \\
\text { abundance score (60), isotope } \\
\text { spacing score (50), retention } \\
\text { time score }(50)\end{array}$ \\
\hline & $\begin{array}{l}\text { Do not match if } \\
\text { score }\end{array}$ & $<30$ \\
\hline \multirow{2}{*}{$\begin{array}{l}\text { Find by Ion - EIC Peak } \\
\text { Integration and Filtering }\end{array}$} & Integration & Agile 2 \\
\hline & Peak height & $>=3000$ counts \\
\hline \multirow{3}{*}{$\begin{array}{l}\text { Find by Ion - Post- } \\
\text { Processing Filters }\end{array}$} & Absolute height & $>=1000$ counts \\
\hline & Score (Tgt) & $>=50$ \\
\hline & $\begin{array}{l}\text { Satisfy Find by } \\
\text { Ion conditions in }\end{array}$ & $>=2$ files \\
\hline
\end{tabular}


Table S5. Internal standard data (as ratio of peak area in sample to peak area in method blank) for (a-b) HRMS and (c-d) LC-MS/MS datasets. Only ISTDs detected via ESI+ are shown for HRMS, and only 9 of 12 ESI+ ISTDs were reliably detected (>50\% of samples); others exhibited poor sensitivity due to low SPE recovery, detector sensitivity, and/or ion suppression.

\begin{tabular}{|c|c|c|c|c|c|c|c|c|c|c|c|c|}
\hline \multirow{2}{*}{$\begin{array}{l}\text { (a) Internal Standard - } \\
\text { HRMS Data }\end{array}$} & \multirow{2}{*}{$\begin{array}{c}\text { July } 26, \\
2018 \\
8: 30 \mathrm{am}- \\
8: 30 \mathrm{pm} \\
\end{array}$} & \multirow{2}{*}{$\begin{array}{c}\text { August } \\
15,2018 \\
7 \mathrm{am}-7 \mathrm{pm} \\
\end{array}$} & \multirow{2}{*}{$\begin{array}{c}\text { Sept 18, } \\
2018 \\
7 \mathrm{am}-7 \mathrm{pm}\end{array}$} & \multicolumn{9}{|c|}{ Storm: October 25-26, 2018} \\
\hline & & & & $\begin{array}{c}10 / 22 \\
11: 50 \mathrm{am} \\
\end{array}$ & $\begin{array}{c}10 / 25 \\
7 \mathrm{am}\end{array}$ & \begin{tabular}{l|c}
5 & $10 / 25$ \\
1 & $2: 20 \mathrm{pm}$ \\
\end{tabular} & $\begin{array}{c}10 / 25 \\
5: 20 \mathrm{pm}\end{array}$ & $\begin{array}{c}10 / 26 \\
12: 30 \mathrm{am} \\
\end{array}$ & \multicolumn{2}{|c|}{$\begin{array}{c}10 / 26 \\
8: 40 \mathrm{am} \\
\end{array}$} & $\begin{array}{c}10 / 26 \\
6: 00 \mathrm{pm} \\
\end{array}$ & $\begin{array}{c}10 / 27 \\
9 \mathrm{am} \\
\end{array}$ \\
\hline Diphenyl-d10-urea & 1.2 & 0.56 & 1.3 & 0.42 & 0.75 & 0.39 & 1.4 & 0.36 & \multicolumn{2}{|c|}{0.41} & 0.44 & 0.17 \\
\hline 5-methyl-1-H-BTR-d6 & 1.2 & 0.88 & 0.70 & 0.64 & 0.54 & 0.90 & 0.91 & 0.74 & \multicolumn{2}{|c|}{0.81} & 0.80 & 0.86 \\
\hline Carbendazim-d4 & 2.0 & 1.4 & 1.1 & 1.2 & 1.3 & 1.8 & 1.9 & 2.1 & \multicolumn{2}{|c|}{2.1} & 2.2 & 1.4 \\
\hline Imidacloprid-d4 & 0.62 & 0.42 & 0.27 & 0.32 & 0.34 & 0.20 & 0.00 & 0.04 & \multicolumn{2}{|c|}{0.08} & 0.10 & 0.25 \\
\hline Cotinine-d3 & 0.58 & 0.56 & 0.52 & 0.70 & 0.67 & 0.62 & 0.43 & 0.44 & \multicolumn{2}{|c|}{0.52} & 0.60 & 0.61 \\
\hline DEET-d7 & 0.81 & 0.91 & 0.91 & 0.76 & 0.82 & 0.72 & 0.23 & 0.24 & \multicolumn{2}{|c|}{0.38} & 0.41 & 0.66 \\
\hline Diuron-d6 & 0.79 & 0.64 & 0.30 & 0.37 & 0.34 & 0.21 & 0.00 & 0.00 & \multicolumn{2}{|c|}{0.09} & 0.13 & 0.22 \\
\hline Prometon-d3 & 1.2 & 0.90 & 0.40 & 0.38 & 0.39 & 0.21 & 0.11 & 0.30 & 0.2 & & 0.24 & 0.20 \\
\hline BEHP-d4 & 1.0 & 1.63 & 3.70 & 0.69 & 0.30 & 0.56 & 0.36 & 0.00 & 0. & & 0.31 & 0.35 \\
\hline Median & 1.0 & 0.88 & 0.70 & 0.64 & 0.5 & 0.56 & 0.36 & 0.30 & 0. & 11 & 0.41 & 0.35 \\
\hline & & m: Noveml & er 2,2018 & & & & orm: Decen & mber $10-1$ & 1,20 & & & \\
\hline $\begin{array}{l}\text { (b) Internal Standard - } \\
\text { HRMS Data }\end{array}$ & $\begin{array}{c}11 / 111 \mathrm{am} \\
-5 \mathrm{pm}\end{array}$ & $\begin{array}{c}11 / 2 \\
3 \mathrm{am}-7 \mathrm{am} \\
\end{array}$ & $\begin{array}{c}11 / 2 \\
7 \mathrm{am}-1 \mathrm{pm} \\
\end{array}$ & $\begin{array}{l}11 / 3 \\
10 \mathrm{am}\end{array}$ & $\begin{array}{l}12 / 10 \\
2 \mathrm{pm}\end{array}$ & $\begin{array}{l}12 / 102 \mathrm{pm}- \\
12 / 112 \mathrm{am}\end{array}$ & $\begin{array}{c}12 / 11 \\
2 \mathrm{am}-8 \mathrm{am}\end{array}$ & $\begin{array}{r}12 / 1 \\
8 \mathrm{am}-2 \\
\end{array}$ & & & $\begin{array}{l}2 / 11 \\
\mathrm{n}-6 \mathrm{pm} \\
\end{array}$ & $\begin{array}{c}12 / 12 \\
2: 30 \mathrm{pm}\end{array}$ \\
\hline Diphenyl-d10-urea & 0.76 & 0.41 & 0.22 & 0.65 & 0.23 & 0.36 & 0.09 & 0.00 & & & 0.00 & 0.48 \\
\hline 5-methyl-1-H-BTR-d6 & 0.88 & 0.95 & 0.95 & 0.79 & 0.27 & 0.41 & 0.53 & 0.53 & & & 0.36 & 0.40 \\
\hline Carbendazim-d4 & 1.5 & 2.1 & 3.4 & 1.6 & 0.90 & 1.1 & 0.84 & 0.84 & & & 1.1 & 1.2 \\
\hline Imidacloprid-d4 & 0.42 & 0.04 & 0.23 & 0.42 & 0.27 & 0.34 & 0.25 & 0.19 & & & 0.19 & 0.52 \\
\hline Cotinine-d3 & 0.58 & 0.45 & 0.51 & 0.64 & 0.70 & 0.70 & 0.70 & 0.55 & & & 0.56 & 0.79 \\
\hline DEET-d7 & 0.79 & 0.68 & 0.67 & 0.67 & 0.17 & 0.23 & 0.19 & 0.19 & & & 0.21 & 0.21 \\
\hline Diuron-d6 & 0.32 & 0.05 & 0.18 & 0.29 & 0.00 & 0.00 & 0.00 & 0.00 & & & 0.00 & 0.00 \\
\hline Prometon-d3 & 0.39 & 0.30 & 0.35 & 0.32 & 0.13 & 0.15 & 0.13 & 0.18 & & & 0.19 & 0.15 \\
\hline BEHP-d4 & 0.70 & 0.30 & 0.37 & 0.59 & 0.25 & 0.26 & 0.28 & 0.00 & & & 0.00 & 0.35 \\
\hline Median & 0.70 & 0.41 & 0.37 & 0.64 & 0.25 & 0.34 & 0.25 & 0.19 & & & 0.19 & 0.40 \\
\hline
\end{tabular}




\begin{tabular}{|c|c|c|c|c|c|c|c|c|c|c|c|}
\hline \multirow{2}{*}{$\begin{array}{c}\text { (c) Internal Standard - } \\
\text { QQQ Data }\end{array}$} & \multirow{2}{*}{$\begin{array}{c}\text { July } 26, \\
2018 \\
8: 30 \mathrm{am}- \\
8: 30 \mathrm{pm}\end{array}$} & \multirow{2}{*}{$\begin{array}{c}\text { August } \\
15,2018 \\
7 \text { am-7pm }\end{array}$} & \multirow{2}{*}{$\begin{array}{c}\begin{array}{c}\text { Sept 18, } \\
2018\end{array} \\
\text { 7am-7pm }\end{array}$} & \multicolumn{8}{|c|}{ Storm: October 25-26, 2018} \\
\hline & & & & $\begin{array}{c}10 / 22 \\
11: 50 \mathrm{am}\end{array}$ & $\begin{array}{c}10 / 25 \\
7 \mathrm{am}\end{array}$ & $\begin{array}{c}10 / 25 \\
2: 20 \mathrm{pm}\end{array}$ & $\begin{array}{c}10 / 25 \\
5: 20 \mathrm{pm}\end{array}$ & $\begin{array}{c}10 / 26 \\
12: 30 \mathrm{am}\end{array}$ & $\begin{array}{c}10 / 26 \\
8: 40 \mathrm{am}\end{array}$ & $\begin{array}{c}10 / 26 \\
6: 00 \mathrm{pm}\end{array}$ & $\begin{array}{c}10 / 27 \\
9 \mathrm{am} \\
\end{array}$ \\
\hline Diphenyl-d10-urea & 0.59 & 1.00 & 0.31 & 0.43 & 0.41 & 0.21 & 0.09 & 0.08 & 0.15 & 0.13 & 0.20 \\
\hline 5-methyl-1-H-BTR-d6 & 0.49 & 0.60 & 0.11 & 0.33 & 0.30 & 0.19 & 0.09 & 0.12 & 0.16 & 0.15 & 0.20 \\
\hline 4-Nitrophenol-d4 & 0.03 & 0.98 & 0.37 & 0.40 & 0.27 & 0.23 & 0.19 & 0.21 & 0.14 & 0.13 & 0.14 \\
\hline Bisphenol A-d8 & 1.16 & 6.36 & 0.80 & 1.02 & 1.78 & 1.32 & 0.89 & 0.99 & 0.62 & 1.39 & 1.56 \\
\hline Caffeine-13C3 & 0.51 & 0.63 & 0.11 & 0.36 & 0.32 & 0.21 & 0.09 & 0.12 & 0.15 & 0.17 & 0.20 \\
\hline Carbendazim-d4 & 0.78 & 0.52 & 0.43 & 0.51 & 0.46 & 0.30 & 0.10 & 0.15 & 0.22 & 0.22 & 0.26 \\
\hline Cetirizin-d8 & 2.05 & 0.66 & 1.3 & 0.85 & 0.72 & 0.31 & 0.13 & 0.15 & 0.21 & 0.22 & 0.28 \\
\hline Imidacloprid-d4 & 0.33 & 0.52 & 0.06 & 0.20 & 0.18 & 0.11 & 0.05 & 0.06 & 0.09 & 0.09 & 0.11 \\
\hline Cotinine-d3 & 0.26 & 0.37 & 0.08 & 0.21 & 0.21 & 0.14 & 0.07 & 0.08 & 0.11 & 0.12 & 0.14 \\
\hline DEET-d7 & 0.74 & 0.78 & 0.54 & 0.67 & 0.64 & 0.41 & 0.21 & 0.22 & 0.32 & 0.33 & 0.38 \\
\hline Diazinon-d10 & 0.82 & 1.1 & 0.86 & 0.88 & 0.92 & 0.88 & 0.66 & 0.60 & 0.79 & 0.67 & 0.68 \\
\hline Diclofenac-d4 & 1.49 & 1.8 & 1.35 & 1.5 & 1.5 & 1.3 & 0.8 & 0.94 & 0.99 & 0.87 & 0.93 \\
\hline Diuron-d6 & 0.62 & 0.94 & 0.37 & 0.49 & 0.43 & 0.23 & 0.09 & 0.10 & 0.15 & 0.17 & 0.22 \\
\hline Prometon-d3 & 0.70 & 0.87 & 0.54 & 0.59 & 0.53 & 0.32 & 0.16 & 0.18 & 0.23 & 0.25 & 0.29 \\
\hline Ibuprofen-d3 & 1.13 & 1.1 & 0.89 & 0.93 & 0.99 & 0.87 & 0.48 & 0.50 & 0.59 & 0.63 & 0.70 \\
\hline Triclosan-d3 & 0.60 & 1.3 & 0.96 & 0.67 & 0.84 & 0.81 & 0.57 & 0.34 & 0.67 & 0.72 & 0.73 \\
\hline BEHP-d4 & 1.54 & 0.38 & 0.50 & 0.53 & 0.92 & 0.74 & 0.57 & 0.43 & 0.73 & 0.46 & 0.38 \\
\hline Median & 0.70 & 0.87 & $\mathbf{0 . 5 0}$ & $\mathbf{0 . 5 3}$ & $\mathbf{0 . 5 3}$ & 0.31 & 0.16 & 0.18 & 0.22 & 0.22 & 0.28 \\
\hline
\end{tabular}




\begin{tabular}{|c|c|c|c|c|c|c|c|c|c|c|}
\hline \multirow[b]{2}{*}{$\begin{array}{c}\text { (d) Internal Standard - } \\
\text { QQQ Data }\end{array}$} & \multicolumn{4}{|c|}{ Storm: November 2, 2018} & \multicolumn{6}{|c|}{ Storm: December 10-11, 2018} \\
\hline & $\begin{array}{c}11 / 1 \\
11 \mathrm{am}-5 \mathrm{pm}\end{array}$ & $\begin{array}{c}11 / 2 \\
3 a m-7 a m\end{array}$ & $\begin{array}{c}11 / 2 \\
7 \mathrm{am}-1 \mathrm{pm}\end{array}$ & $\begin{array}{l}11 / 3 \\
10 \mathrm{am}\end{array}$ & $\begin{array}{l}12 / 10 \\
2 \mathrm{pm}\end{array}$ & $\begin{array}{l}12 / 102 \mathrm{pm}- \\
12 / 112 \mathrm{am}\end{array}$ & $\begin{array}{c}12 / 11 \\
2 \mathrm{am}-8 \mathrm{am}\end{array}$ & $\begin{array}{c}12 / 11 \\
8 \mathrm{am}-2 \mathrm{pm}\end{array}$ & $\begin{array}{c}12 / 11 \\
2 \mathrm{pm}-6 \mathrm{pm}\end{array}$ & $\begin{array}{c}12 / 12 \\
2: 30 \mathrm{pm}\end{array}$ \\
\hline Diphenyl-d10-urea & 0.23 & 0.12 & 0.15 & 0.19 & 0.25 & 0.31 & 0.27 & 0.24 & 0.28 & 0.42 \\
\hline 5-methyl-1-H-BTR-d6 & 0.14 & 0.10 & 0.11 & 0.13 & 0.10 & 0.09 & 0.08 & 0.09 & 0.11 & 0.10 \\
\hline 4-Nitrophenol-d4 & 0.79 & 0.64 & 0.76 & 0.76 & 0.05 & 0.01 & 0.08 & 0.17 & 0.40 & 0.31 \\
\hline Bisphenol A-d8 & 0.66 & 0.97 & 1.39 & 0.52 & 0.37 & 0.15 & 0.41 & 0.95 & 0.26 & 1.55 \\
\hline Caffeine-13C 3 & 0.23 & 0.17 & 0.21 & 0.22 & 0.12 & 0.12 & 0.12 & 0.12 & 0.13 & 0.14 \\
\hline Carbendazim-d4 & 0.12 & 0.08 & 0.09 & 0.11 & 0.13 & 0.14 & 0.13 & 0.14 & 0.17 & 0.16 \\
\hline Cetirizin-d8 & 0.18 & 0.12 & 0.15 & 0.17 & 0.54 & 0.53 & 0.58 & 0.53 & 0.57 & 0.74 \\
\hline Imidacloprid-d4 & 0.11 & 0.07 & 0.09 & 0.11 & 0.05 & 0.05 & 0.05 & 0.05 & 0.06 & 0.07 \\
\hline Cotinine-d3 & 0.11 & 0.08 & 0.09 & 0.10 & 0.06 & 0.06 & 0.06 & 0.06 & 0.07 & 0.07 \\
\hline DEET-d7 & 0.52 & 0.35 & 0.41 & 0.49 & 0.64 & 0.70 & 0.66 & 0.60 & 0.63 & 0.87 \\
\hline Diazinon-d10 & 1.0 & 0.81 & 0.96 & 1.1 & 1.4 & 1.4 & 1.3 & 1.3 & 1.3 & 1.7 \\
\hline Diclofenac-d4 & 1.0 & 0.82 & 0.94 & 1.0 & 1.7 & 1.4 & 1.6 & 1.5 & 1.4 & 1.5 \\
\hline Diuron-d6 & 0.27 & 0.15 & 0.19 & 0.25 & 0.33 & 0.37 & 0.33 & 0.30 & 0.36 & 0.47 \\
\hline Prometon-d3 & 0.44 & 0.27 & 0.32 & 0.41 & 0.37 & 0.44 & 0.37 & 0.35 & 0.39 & 0.55 \\
\hline Ibuprofen-d3 & 0.79 & 0.70 & 0.70 & 0.73 & 0.91 & 0.87 & 0.88 & 0.90 & 0.92 & 0.93 \\
\hline Triclosan-d3 & 0.95 & 0.67 & 0.84 & 0.94 & 1.0 & 0.82 & 0.92 & 0.93 & 1.1 & 1.1 \\
\hline BEHP-d4 & 0.41 & 0.07 & 0.37 & 0.34 & 0.60 & 0.44 & 0.56 & 0.45 & 0.55 & 0.71 \\
\hline Median & 0.41 & 0.17 & 0.32 & 0.34 & 0.37 & 0.37 & 0.37 & 0.35 & 0.39 & 0.55 \\
\hline
\end{tabular}


Table S6. Concentrations of targeted organic contaminants quantified via LC/MS/MS analysis in Miller Creek during (a) late summer baseflow conditions and (b-d) fall storm events of 2018. Units are $\mathrm{ng} / \mathrm{L}$ for all values, with relative standard deviation (\% RSD) in parenthesis. ND = non-detect, $\mathrm{BQ}=$ between MDL and MQL. MDL and MQL values provided in Table S2. Analytes marked with an asterisk $(*)$ are semi-quantitative, as only one ion transition was monitored.

\begin{tabular}{|c|c|c|c|c|}
\hline (a) & Sampling Date: & July 26,2018 & Aug. 15, 2018 & Sept. 18, 2018 \\
\hline & Event Type: & & Baseflow & \\
\hline $\begin{array}{l}\text { Contaminant } \\
\text { Group }\end{array}$ & Compound & $\begin{array}{l}\text { 8:30am- } \\
8: 30 \mathrm{pm}\end{array}$ & $7 \mathrm{am}-7 \mathrm{pm}$ & $7 \mathrm{am}-7 \mathrm{pm}$ \\
\hline & 1,3-Diphenylguanidine & $17.5 \quad(2 \%)$ & $(2 \%)$ & $(7 \%)$ \\
\hline derived & HMMM & $1.3 \quad(17 \%)$ & $(5 \%)$ & $(5 \%)$ \\
\hline & $\mathrm{NCBA}$ & $0.6 \quad(32 \%)$ & $(5 \%)$ & $(0.3 \%)$ \\
\hline & Benzotriazole & $22.8 \quad(26 \%)$ & $(6 \%)$ & $(2 \%)$ \\
\hline & 5-methylbenzotriazole & $43.6 \quad(25 \%)$ & $(5 \%)$ & $(2 \%)$ \\
\hline BTH / BTR & 2-amino-benzothiazole & $1.8 \quad(21 \%)$ & $(8 \%)$ & $(6 \%)$ \\
\hline & 2-hydroxy-benzothiazole (2-OH-BTH) & $73.5 \quad(20 \%)$ & $(3 \%)$ & $(19 \%)$ \\
\hline & 2-(4-morpholinyl)-benzothiazole (2,4-MoBT) & $5.0 \quad(19 \%)$ & ND & $11.1 \quad(4 \%)$ \\
\hline & 1,3-Dicyclohexylurea & $4.6 \quad(33 \%)$ & $(5 \%)$ & $56.1 \quad(1.5 \%)$ \\
\hline Industrial & Bisphenol A & ND & ND & ND \\
\hline Chemicals & 4-Nonylphenol* & ND & ND & ND \\
\hline & 4-tert-Octylphenol & ND & ND & ND \\
\hline & Clothianidin & ND & ND & ND \\
\hline & Imidacloprid & ND & $(5 \%)$ & $(5 \%)$ \\
\hline & Thiamethoxam & ND & ND & ND \\
\hline & Fipronil & $0.1 \quad(33 \%)$ & $(6 \%)$ & ND \\
\hline Pesticides & Carbendazim & ND & $(2 \%)$ & $(2 \%)$ \\
\hline 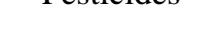 & Pentachlorophenol & $32.2 \quad(36 \%)$ & $21.1 \quad(3 \%)$ & $(8 \%)$ \\
\hline & Diazinon & ND & ND & $(1.4 \%)$ \\
\hline & Diuron & $4.4 \quad(28 \%)$ & $(6 \%)$ & $(9 \%)$ \\
\hline & Mecoprop & ND & ND & $(8 \%)$ \\
\hline & Prometon & $(29 \%)$ & $(8 \%)$ & $(5 \%)$ \\
\hline
\end{tabular}




\begin{tabular}{|c|c|c|c|c|c|c|c|}
\hline \multirow{7}{*}{ PPCPs } & Caffeine & 3.7 & $(38 \%)$ & 3.6 & $(11 \%)$ & 55.7 & $(5 \%)$ \\
\hline & Cetirizine & 0.5 & $(3 \%)$ & 1.4 & $(1 \%)$ & 0.6 & $(6 \%)$ \\
\hline & Cotinine & 1.1 & $(38 \%)$ & 2.1 & $(4 \%)$ & 6.8 & $(0.8 \%)$ \\
\hline & DEET & 6.3 & $(35 \%)$ & 10.7 & $(4 \%)$ & 17.0 & $(5 \%)$ \\
\hline & Diclofenac & \multicolumn{2}{|c|}{ ND } & \multicolumn{2}{|c|}{ ND } & \multicolumn{2}{|c|}{ ND } \\
\hline & Ibuprofen* & \multicolumn{2}{|c|}{$\mathrm{ND}$} & \multicolumn{2}{|c|}{ ND } & \multicolumn{2}{|c|}{ ND } \\
\hline & Triclosan* & \multicolumn{2}{|c|}{ ND } & \multicolumn{2}{|c|}{$\mathrm{ND}$} & \multicolumn{2}{|c|}{ ND } \\
\hline \multirow{6}{*}{$\begin{array}{c}\text { SDPA/BZT- } \\
\text { UV }\end{array}$} & SDPA-diAMS & 0.9 & $(44 \%)$ & 0.6 & $(30 \%)$ & 0.5 & $(22 \%)$ \\
\hline & SDPA-C4C8 & 1.5 & $(9 \%)$ & 2.5 & $(5 \%)$ & 1.7 & $(6 \%)$ \\
\hline & SDPA-C8C8* & 4.8 & $(47 \%)$ & 7.1 & $(26 \%)$ & 6.0 & $(24 \%)$ \\
\hline & SDPA-C9C9* & \multicolumn{2}{|c|}{ ND } & 16.0 & $(23 \%)$ & \multicolumn{2}{|c|}{ ND } \\
\hline & BZT\&UV-234 & \multicolumn{2}{|c|}{$\mathrm{ND}$} & 0.5 & $(3 \%)$ & 2.7 & $(66 \%)$ \\
\hline & BZT\&UV-326 & \multicolumn{2}{|c|}{$\mathrm{ND}$} & \multicolumn{2}{|c|}{ ND } & \multicolumn{2}{|c|}{ ND } \\
\hline
\end{tabular}

\begin{tabular}{|c|c|c|c|c|c|c|c|c|c|}
\hline \multirow{2}{*}{\multicolumn{2}{|c|}{$\begin{array}{rr}\text { (b) } & \text { Sampling Date: } \\
\end{array}$}} & \multicolumn{8}{|c|}{ October 25-27, 2018} \\
\hline & & \multicolumn{8}{|c|}{ Storm } \\
\hline \multirow{2}{*}{$\begin{array}{c}\begin{array}{c}\text { Contaminant } \\
\text { Group }\end{array} \\
\end{array}$} & \multirow{2}{*}{$\begin{array}{c}\text { Compound } \\
\text { 1,3-Diphenylguanidine }\end{array}$} & $\begin{array}{c}10 / 22 \\
11: 50 \mathrm{am}\end{array}$ & $10 / 257 \mathrm{am}$ & $\begin{array}{c}10 / 25 \\
2: 20 \mathrm{pm}\end{array}$ & $\begin{array}{c}10 / 25 \\
5: 20 \mathrm{pm}\end{array}$ & 10/26 12:30am & $\begin{array}{c}10 / 26 \\
8: 40 \mathrm{am}\end{array}$ & $\begin{array}{c}10 / 26 \\
6: 00 \mathrm{pm}\end{array}$ & 10/27 9am \\
\hline & & $26.8 \quad(3 \%)$ & $79.9 \quad(1 \%)$ & $(6 \%)$ & $(2 \%)$ & $(1 \%)$ & $(8 \%)$ & $(2 \%)$ & $96.7 \quad(2 \%)$ \\
\hline $\mathrm{v}$ & HMMM & $19.3 \quad(2 \%)$ & $43.1 \quad(3 \%)$ & $(2 \%)$ & $978 \quad(10 \%)$ & $(9 \%)$ & $(4 \%)$ & $(2 \%)$ & $(8 \%)$ \\
\hline & NCBA & $(9 \%)$ & BQ $\quad(15 \%)$ & $(6 \%)$ & $(3 \%)$ & $23.5 \quad(11 \%)$ & $11.6 \quad(7 \%)$ & $(5 \%)$ & $(4 \%)$ \\
\hline \multirow{5}{*}{$\mathrm{BTH} / \mathrm{BTR}$} & Benzotriazole & $26.1 \quad(4 \%)$ & $61.4 \quad(3 \%)$ & $(7 \%)$ & $(1 \%)$ & $(7 \%)$ & $(7 \%)$ & $(9 \%)$ & $294 \quad(10 \%)$ \\
\hline & 5-methylbenzotriazole & $47.0 \quad(2 \%)$ & $97.8 \quad(2 \%)$ & $(4 \%)$ & $(2 \%)$ & $(4 \%)$ & $341 \quad(14 \%)$ & $(6 \%)$ & $(2 \%)$ \\
\hline & 2-amino-benzothiazole & $2.4 \quad(11 \%)$ & $(4 \%)$ & $(5 \%)$ & $22.3 \quad(11 \%)$ & $(10 \%)$ & $(7 \%)$ & $(8 \%)$ & $(2 \%)$ \\
\hline & 2-OH-BTH & $42.0 \quad(6 \%)$ & $76.8 \quad(3 \%)$ & $242 \quad(11 \%)$ & $(9 \%)$ & $(10 \%)$ & $(3 \%)$ & $263 \quad(11 \%)$ & $267 \quad(14 \%)$ \\
\hline & 2,4-MoBT & ND & $(4 \%)$ & $9.9 \quad(14 \%)$ & $(5 \%)$ & $(12 \%)$ & $18.3 \quad(0 \%)$ & $17.3 \quad(2 \%)$ & $10.2 \quad(9 \%)$ \\
\hline \multirow{4}{*}{$\begin{array}{l}\text { Industrial } \\
\text { Chemicals }\end{array}$} & 1,3-Dicyclohexylurea & $(6 \%)$ & $21.3 \quad(2 \%)$ & $128 \quad(6 \%)$ & $(6 \%)$ & $(7 \%)$ & $155 \quad(6 \%)$ & $147 \quad(3 \%)$ & $91.9 \quad(8 \%)$ \\
\hline & Bisphenol A & ND & ND & ND & ND & $88.7 \quad(16 \%)$ & ND & ND & ND \\
\hline & 4-Nonylphenol* & ND & ND & ND & ND & ND & ND & ND & ND \\
\hline & 4-tert-Octylphenol & ND & ND & ND & ND & ND & ND & ND & ND \\
\hline
\end{tabular}




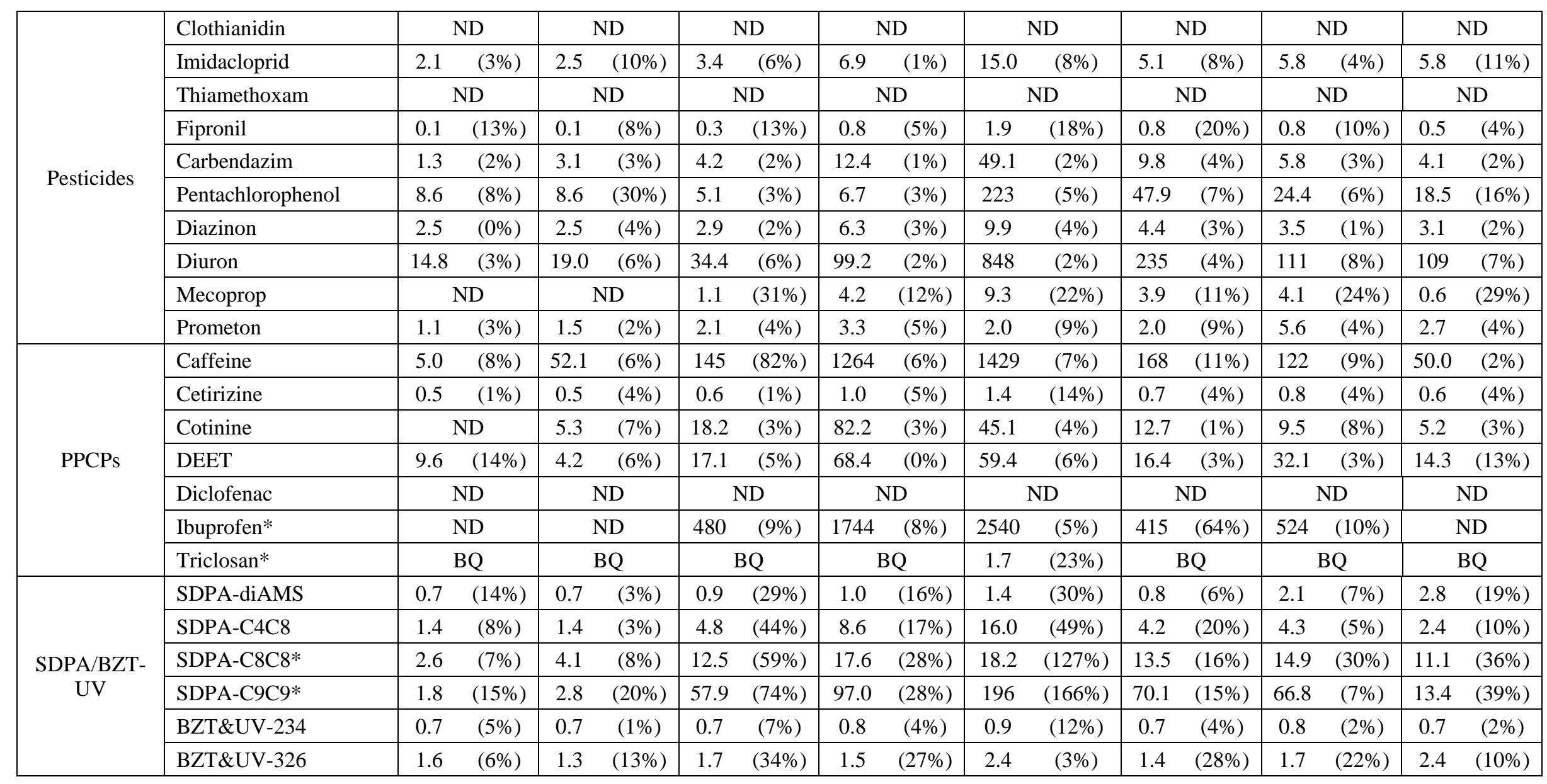




\begin{tabular}{|c|c|c|c|c|c|}
\hline \multirow{2}{*}{\multicolumn{2}{|c|}{$\begin{array}{rr}\text { (c) } & \text { Sampling Date: } \\
\end{array}$}} & \multicolumn{4}{|c|}{ November 1-3, 2018} \\
\hline & & \multicolumn{4}{|c|}{ Storm } \\
\hline $\begin{array}{l}\text { Contaminant } \\
\text { Group }\end{array}$ & Compound & $\begin{array}{l}\text { 11/1 11am- } \\
5 \mathrm{pm}\end{array}$ & 11/2 3am-7am & $\begin{array}{l}11 / 27 \mathrm{am}- \\
1 \mathrm{pm}\end{array}$ & 11/3 10am \\
\hline \multirow{3}{*}{$\begin{array}{l}\text { Vehicle- } \\
\text { derived }\end{array}$} & 1,3-Diphenylguanidine & $37.1 \quad(10 \%)$ & $(4 \%)$ & $359 \quad(5 \%)$ & $(2 \%)$ \\
\hline & HMMM & $88.8 \quad(9 \%)$ & $(3 \%)$ & $180 \quad(4 \%)$ & $(5 \%)$ \\
\hline & NCBA & $2.3 \quad(13 \%)$ & $(7 \%)$ & $(4 \%)$ & $(11 \%)$ \\
\hline \multirow{5}{*}{ BTH / BTR } & Benzotriazole & $100 \quad(1 \%)$ & $(2 \%)$ & $190 \quad(4 \%)$ & $138 \quad(11 \%)$ \\
\hline & 5-methylbenzotriazole & $145 \quad(9 \%)$ & $(1 \%)$ & $340 \quad(4 \%)$ & $(10 \%)$ \\
\hline & 2-amino-benzothiazole & $5.5 \quad(14 \%)$ & $22.3 \quad(26 \%)$ & $10.7 \quad(6 \%)$ & $(7 \%)$ \\
\hline & 2-OH-BTH & $109 \quad(14 \%)$ & $(2 \%)$ & $403 \quad(11 \%)$ & $(9 \%)$ \\
\hline & 2,4-MoBT & $7.1 \quad(9 \%)$ & $(5 \%)$ & $13.4 \quad(6 \%)$ & $(4 \%)$ \\
\hline \multirow{4}{*}{$\begin{array}{l}\text { Industrial } \\
\text { Chemicals }\end{array}$} & 1,3-Dicyclohexylurea & $27.7 \quad(5 \%)$ & $(4 \%)$ & $80.9 \quad(6 \%)$ & $29.4 \quad(5 \%)$ \\
\hline & Bisphenol A & ND & $52.3 \quad(20 \%)$ & ND & ND \\
\hline & 4-Nonylphenol* & ND & ND & ND & ND \\
\hline & 4-tert-Octylphenol & ND & ND & ND & ND \\
\hline \multirow{10}{*}{ Pesticides } & Clothianidin & ND & $(8 \%)$ & $2.6 \quad(3 \%)$ & ND \\
\hline & Imidacloprid & $4.3 \quad(3 \%)$ & $(4 \%)$ & $(7 \%)$ & $(1 \%)$ \\
\hline & Thiamethoxam & $\mathrm{ND}$ & ND & ND & ND \\
\hline & Fipronil & $0.5 \quad(6 \%)$ & $(14 \%)$ & $0.8 \quad(2 \%)$ & $(9 \%)$ \\
\hline & Carbendazim & $2.7 \quad(2 \%)$ & $(1 \%)$ & $12.2 \quad(6 \%)$ & $(8 \%)$ \\
\hline & Pentachlorophenol & $21.0 \quad(23 \%)$ & $(18 \%)$ & $83.3 \quad(13 \%)$ & $28.6 \quad(12 \%)$ \\
\hline & Diazinon & $1.1 \quad(2 \%)$ & $(1 \%)$ & $(3 \%)$ & $(1 \%)$ \\
\hline & Diuron & $34.4 \quad(4 \%)$ & $(3 \%)$ & $173 \quad(1 \%)$ & $62.8 \quad(7 \%)$ \\
\hline & Mecoprop & ND & ND & ND & ND \\
\hline & Prometon & $1.5 \quad(9 \%)$ & $(1 \%)$ & $(4 \%)$ & $(5 \%)$ \\
\hline \multirow{4}{*}{ PPCPs } & Caffeine & $32.0 \quad(4 \%)$ & $(4 \%)$ & $158 \quad(12 \%)$ & $(9 \%)$ \\
\hline & Cetirizine & $0.4 \quad(4 \%)$ & $(3 \%)$ & $(4 \%)$ & $(9 \%)$ \\
\hline & Cotinine & $5.4 \quad(2 \%)$ & $(2 \%)$ & $12.8 \quad(3 \%)$ & $(11 \%)$ \\
\hline & DEET & $22.1 \quad(5 \%)$ & $(2 \%)$ & $19.7 \quad(2 \%)$ & $6.4 \quad(11 \%)$ \\
\hline
\end{tabular}




\begin{tabular}{|c|c|c|c|c|c|}
\hline \multirow{3}{*}{ PPCPs } & Diclofenac & ND & ND & \multirow{2}{*}{$\begin{array}{l}\mathrm{ND} \\
\mathrm{ND}\end{array}$} & \multirow{2}{*}{$\begin{array}{l}\text { ND } \\
\text { ND }\end{array}$} \\
\hline & Ibuprofen* & ND & $1065 \quad(6 \%)$ & & \\
\hline & Triclosan* & ND & ND & ND & ND \\
\hline \multirow{6}{*}{$\begin{array}{c}\text { SDPA/BZT- } \\
\text { UV }\end{array}$} & SDPA-diAMS & $1.0 \quad(22 \%)$ & $0.4 \quad(173 \%)$ & $1.0 \quad(8 \%)$ & $0.4 \quad(88 \%)$ \\
\hline & SDPA-C4C8 & $1.6 \quad(10 \%)$ & $(96 \%)$ & $(9 \%)$ & $(8 \%)$ \\
\hline & SDPA-C8C8* & $3.4 \quad(15 \%)$ & ND & $3.7 \quad(26 \%)$ & $4.6 \quad(31 \%)$ \\
\hline & SDPA-C9C9* & ND & ND & $13.3 \quad(24 \%)$ & ND \\
\hline & BZT\&UV-234 & $0.6 \quad(5 \%)$ & $0.8 \quad(31 \%)$ & $0.6 \quad(6 \%)$ & $0.2 \quad(173 \%)$ \\
\hline & BZT\&UV-326 & ND & ND & ND & ND \\
\hline
\end{tabular}

\begin{tabular}{|c|c|c|c|c|c|c|c|}
\hline \multirow{2}{*}{\multicolumn{2}{|c|}{$\begin{array}{ll}\text { (d) } & \text { Sampling Date: } \\
\end{array}$}} & \multicolumn{6}{|c|}{ December 10-12, 2018} \\
\hline & & \multicolumn{6}{|c|}{ Storm } \\
\hline $\begin{array}{l}\text { Contaminant } \\
\text { Group }\end{array}$ & Compound & $12 / 102 \mathrm{pm}$ & $\begin{array}{l}12 / 102 \mathrm{pm}- \\
12 / 112 \mathrm{am}\end{array}$ & $\begin{array}{l}\text { 12/11 2am- } \\
\quad 8 \mathrm{am}\end{array}$ & $\begin{array}{l}12 / 118 \mathrm{am}- \\
2 \mathrm{pm}\end{array}$ & $\begin{array}{l}12 / 112 \mathrm{pm}- \\
\quad 6 \mathrm{pm}\end{array}$ & $12 / 122: 30 \mathrm{pm}$ \\
\hline \multirow{3}{*}{$\begin{array}{l}\text { Vehicle- } \\
\text { derived }\end{array}$} & 1,3-Diphenylguanidine & $546 \quad(7 \%)$ & $254 \quad(3 \%)$ & $449 \quad(12 \%)$ & $639 \quad(6 \%)$ & $640 \quad(5 \%)$ & $(2 \%)$ \\
\hline & HMMM & $480 \quad(4 \%)$ & $290 \quad(8 \%)$ & $366 \quad(6 \%)$ & $612 \quad(7 \%)$ & $631 \quad(4 \%)$ & $(6 \%)$ \\
\hline & NCBA & $28.0 \quad(1 \%)$ & $20.5 \quad(5 \%)$ & $30.8 \quad(5 \%)$ & $39.2 \quad(4 \%)$ & $45.6 \quad(4 \%)$ & $(4 \%)$ \\
\hline \multirow{5}{*}{$\mathrm{BTH} / \mathrm{BTR}$} & Benzotriazole & $163 \quad(3 \%)$ & $153 \quad(6 \%)$ & $332 \quad(9 \%)$ & $373 \quad(16 \%)$ & $168 \quad(6 \%)$ & $(6 \%)$ \\
\hline & 5-methylbenzotriazole & $414 \quad(9 \%)$ & $295 \quad(2 \%)$ & $542 \quad(2 \%)$ & $635 \quad(9 \%)$ & $508 \quad(10 \%)$ & $(2 \%)$ \\
\hline & 2-amino-benzothiazole & $8.7 \quad(3 \%)$ & $9.2 \quad(4 \%)$ & $10.7 \quad(7 \%)$ & $11.7 \quad(9 \%)$ & $11.6 \quad(1 \%)$ & $(2 \%)$ \\
\hline & 2-OH-BTH & $263 \quad(4 \%)$ & $240 \quad(5 \%)$ & $354 \quad(10 \%)$ & $456 \quad(6 \%)$ & $461 \quad(14 \%)$ & $137 \quad(14 \%)$ \\
\hline & 2,4-MoBT & $31.9 \quad(2 \%)$ & $25.0 \quad(11 \%)$ & $35.5 \quad(5 \%)$ & $48.8 \quad(10 \%)$ & $56.3 \quad(4 \%)$ & $(4 \%)$ \\
\hline \multirow{4}{*}{$\begin{array}{l}\text { Industrial } \\
\text { Chemicals }\end{array}$} & 1,3-Dicyclohexylurea & $175 \quad(5 \%)$ & $91.3 \quad(10 \%)$ & $166 \quad(6 \%)$ & $223 \quad(6 \%)$ & $158 \quad(3 \%)$ & $42.2 \quad(6 \%)$ \\
\hline & Bisphenol A & ND & ND & ND & ND & ND & ND \\
\hline & 4-Nonylphenol* & ND & ND & ND & ND & ND & ND \\
\hline & 4-tert-Octylphenol & ND & ND & ND & ND & ND & ND \\
\hline \multirow{3}{*}{ Pesticides } & Clothianidin & ND & ND & ND & ND & ND & ND \\
\hline & Imidacloprid & $4.9 \quad(12 \%)$ & $4.6 \quad(20 \%)$ & $4.4 \quad(6 \%)$ & $4.5 \quad(8 \%)$ & $4.5 \quad(10 \%)$ & $(4 \%)$ \\
\hline & Thiamethoxam & ND & ND & ND & ND & ND & ND \\
\hline
\end{tabular}




\begin{tabular}{|c|c|c|c|c|c|c|c|c|}
\hline \multirow{7}{*}{ Pesticides } & Fipronil & $(5 \%)$ & $0.5 \quad(14 \%)$ & $(9 \%)$ & $(9 \%)$ & $0.9 \quad(10 \%)$ & 0.4 & $(12 \%)$ \\
\hline & Carbendazim & $4.2 \quad(2 \%)$ & $2.8 \quad(3 \%)$ & $(3 \%)$ & $12.3 \quad(3 \%)$ & $11.3(1 \%)$ & 2.0 & $(2 \%)$ \\
\hline & Pentachlorophenol & $46.8 \quad(18 \%)$ & $42.5 \quad(12 \%)$ & $42.0 \quad(9 \%)$ & $89.9 \quad(12 \%)$ & $(5 \%)$ & 37.2 & $(4 \%)$ \\
\hline & Diazinon & $(6 \%)$ & $(5 \%)$ & $(1 \%)$ & $(5 \%)$ & $(1 \%)$ & 0.8 & $(7 \%)$ \\
\hline & Diuron & $75.2 \quad(3 \%)$ & $36.4 \quad(4 \%)$ & $(3 \%)$ & $(2 \%)$ & $(3 \%)$ & 33.3 & $(3 \%)$ \\
\hline & Mecoprop & $\mathrm{BQ}$ & ND & ND & $1.3 \quad(35 \%)$ & $1.8 \quad(16 \%)$ & 0.4 & $(112 \%)$ \\
\hline & Prometon & $(1 \%)$ & $(0 \%)$ & $(3 \%)$ & $(2 \%)$ & $(2 \%)$ & 1.9 & $(5 \%)$ \\
\hline \multirow{7}{*}{ PPCPs } & Caffeine & $(3 \%)$ & $(9 \%)$ & $(2 \%)$ & $(4 \%)$ & $(3 \%)$ & 51.1 & $(8 \%)$ \\
\hline & Cetirizine & $(1 \%)$ & $(1 \%)$ & $(0 \%)$ & $(1 \%)$ & $(0 \%)$ & 2.1 & $(0 \%)$ \\
\hline & Cotinine & $14.5 \quad(3 \%)$ & $(2 \%)$ & $(2 \%)$ & $(3 \%)$ & $(2 \%)$ & 4.3 & $(8 \%)$ \\
\hline & DEET & $10.0 \quad(1 \%)$ & $18.8 \quad(3 \%)$ & $19.1 \quad(2 \%)$ & $20.7 \quad(4 \%)$ & $22.8 \quad(1 \%)$ & & ND \\
\hline & Diclofenac & ND & ND & ND & ND & ND & \multicolumn{2}{|c|}{ ND } \\
\hline & Ibuprofen* & $620 \quad(5 \%)$ & $(27 \%)$ & $(8 \%)$ & $535 \quad(12 \%)$ & $496 \quad(14 \%)$ & & ND \\
\hline & Triclosan* & ND & ND & ND & ND & ND & \multicolumn{2}{|c|}{ ND } \\
\hline \multirow{6}{*}{$\begin{array}{c}\text { SDPA/BZT- } \\
\text { UV }\end{array}$} & SDPA-diAMS & $0.5 \quad(29 \%)$ & $0.5 \quad(43 \%)$ & $0.9 \quad(23 \%)$ & $1.4 \quad(17 \%)$ & $1.4 \quad(20 \%)$ & 0.6 & $(14 \%)$ \\
\hline & SDPA-C4C8 & $6.6 \quad(32 \%)$ & $(8 \%)$ & $6.8 \quad(13 \%)$ & $16.9 \quad(23 \%)$ & $42.7 \quad(32 \%)$ & 2.7 & $(15 \%)$ \\
\hline & SDPA-C $8 \mathrm{C} 8 *$ & $10.2(19 \%)$ & $(17 \%)$ & $10.7 \quad(30 \%)$ & $21.5 \quad(11 \%)$ & $33.5 \quad(16 \%)$ & 18.7 & $(32 \%)$ \\
\hline & SDPA-C9C9* & $34.8 \quad(18 \%)$ & $10.8 \quad(23 \%)$ & $54.4 \quad(18 \%)$ & $(4 \%)$ & $(8 \%)$ & 7.8 & $(20 \%)$ \\
\hline & BZT\&UV-234 & $(5 \%)$ & $(3 \%)$ & $(2 \%)$ & $(5 \%)$ & $(12 \%)$ & 0.5 & $(2 \%)$ \\
\hline & BZT\&UV-326 & $(21 \%)$ & $(5 \%)$ & $(38 \%)$ & $(5 \%)$ & $(12 \%)$ & 1.2 & $(89 \%)$ \\
\hline
\end{tabular}


Estimated Contaminant Source Strength. To back-calculate the necessary contaminant source strength to produce the observed total concentration in the 10/25/18 5:20pm sample, we used a simple mass-balance approach (Equation S1). We assumed that the flow rate of the "source" was the difference between the observed discharge at 5:20pm and the flow rate during pre-storm baseflow conditions, and that the final concentration was a linear combination of the baseflow and source contributions.

$$
\begin{gathered}
C_{\text {source }} \times Q_{\text {source }}+C_{\text {baseflow }} \times Q_{\text {baseflow }}=C_{\text {combined }} \times Q_{\text {combined }} \\
C_{\text {source }} \times\left(0.014 \mathrm{~m}^{3} / \mathrm{s}\right)+500 \mathrm{ng} / \mathrm{L} \times\left(0.036 \mathrm{~m}^{3} / \mathrm{s}\right) \\
=8220 \mathrm{ng} / \mathrm{L} \times\left(0.05 \mathrm{~m}^{3} / \mathrm{s}\right)
\end{gathered}
$$

\section{Equation S1}

DPG, HMMM, and DCU Loading in TWP. The concentrations of DPG, HMMM, and DCU in TWP leachate ( $24 \mathrm{~h}$ batch leaching, ambient temperature) generated at 1, 5, 15, 65, and 250 $\mathrm{mg} / \mathrm{L}$ were quantified by LC-MS/MS (with extract dilution as needed for concentrations above instrument linear range). Assuming that $100 \%$ of water-leachable contaminant was released, concentrations were converted to per-mass loadings via the known TWP concentrations by Equation S2. We note that the DPG loading calculated here (101 $\mu \mathrm{g}$ DPG / g TWP) aligned well with a previous report of water-leachable DPG in un-aged tire and road wear particles (TRWP). ${ }^{3}$ Assuming a 1:1 ratio of tread to road-derived minerals in TRWP, ${ }^{4}$ the previously reported value of $47 \mu \mathrm{g}$ DPG / $\mathrm{g}$ TRWP is equivalent to $94 \mu \mathrm{g}$ DPG / $\mathrm{g}$ TWP. ${ }^{3}$

$$
\frac{\mu g \text { contaminant }}{g T W P}=\frac{\frac{n g \text { contaminant }}{L \text { water }} \times \frac{\mu g}{1000 n g}}{\frac{m g T W P}{L \text { water }} \times \frac{g}{1000 m g}}
$$

Equation S2

Table S7. DPG, HMMM, and DCU concentrations in TWP leachate (generated at different, known TWP concentrations) and corresponding contaminant loading in TWP.

\begin{tabular}{|c|c|c|c|c|c|c|}
\hline \multirow{2}{*}{$\begin{array}{c}\text { TWP } \\
\text { Conc. } \\
(\mathbf{m g} / \mathbf{L})\end{array}$} & $\begin{array}{c}\text { Leachate } \\
\text { Concentration } \\
(\mathbf{n g} / \mathbf{L})\end{array}$ & $\begin{array}{c}\text { Loading } \\
\text { in TWP } \\
(\boldsymbol{\mu g} / \mathbf{g})\end{array}$ & $\begin{array}{c}\text { Leachate } \\
\text { Concentration } \\
(\mathbf{n g} / \mathbf{L})\end{array}$ & $\begin{array}{c}\text { Loading in } \\
\mathbf{T W P} \\
(\boldsymbol{\mu g} / \mathbf{g})\end{array}$ & $\begin{array}{c}\text { Leachate } \\
\text { Concentration } \\
(\mathbf{n g} / \mathbf{L})\end{array}$ & $\begin{array}{c}\text { Loading in } \\
\text { TWP } \\
(\boldsymbol{\mu g} / \mathbf{g})\end{array}$ \\
\hline 1 & 99 & 99 & 24 & 24 & 20 & 20 \\
\hline 5 & 370 & 74 & 114 & 23 & 79 & 16 \\
\hline 15 & 1660 & 111 & 386 & 26 & 290 & 19 \\
\hline 65 & 7070 & 109 & 1590 & 24 & 1030 & 16 \\
\hline 250 & 28400 & 114 & 4750 & 19 & 5130 & 21 \\
\hline & Average: & $\mathbf{1 0 1} \mathbf{1 6}$ & Average: & $\mathbf{2 3} \pm \mathbf{3}$ & Average: & $\mathbf{1 8} \pm \mathbf{2}$ \\
\hline
\end{tabular}


TWP Concentration Estimates. In addition to estimates of TWP concentration made via HRMS, ${ }^{5}$ we also converted concentrations of DPG, HMMM, and DCU (quantified via LCMS/MS) to TWP concentration, using the loading data shown above. The results are shown in Table 3 of the main text and Figure S7 below. These TWP concentrations again reflected the relative quantity of TWPs needed to explain the observed abundance of each contaminant in Miller Creek. Further, we also used the minimum and maximum water-leachable DPG concentrations in TRWP reported by Unice et al. (94 and $16.2 \mu \mathrm{g}$ DPG/g TWP, based on values of 47 and $8.1 \mu \mathrm{g}$ DPG / g TRWP).,

Unice et al. reported a factor of $\sim 10$ difference in total DPG content and a factor of $\sim 6$ difference in water-leachable DPG content for differentially aged TRWP. ${ }^{3}$ As expected, the DPG-based estimates for TWP concentration made here differed by a factor of $\sim 6$ (i.e., $\sim 6$ times more mass of aged TWP than un-aged TWP was required to produce the same concentration of DPG in the receiving water).

Given that the leachable DPG levels quantified in the TWP used here were equivalent to the leachable DPG levels in un-aged TRWP, we anticipate that both HMMM and DCU levels are lower in aged TRWP. Therefore, the TWP estimates made based on HMMM and DCU concentrations may actually underestimate TWP concentrations.

Notably, the majority of HRMS-based estimates were derived from a new method for source quantification and tracking based upon unidentified non-target HRMS data ${ }^{5}$ collected for hundreds to thousands of feature detections in these complex chemical mixtures. To date, this method has limited environmental validation and application outside of our laboratory. Despite this uncertainty, estimates derived from the HRMS non-target data set were within 1-10-fold of estimates made based on individual contaminant concentrations derived from LC-MS/MS analyses and also broadly agreed with values in the published literature from other studies. ${ }^{6-11}$ Given that the HRMS-based estimates represented the environmental abundance of a broad suite of TWP-derived chemical contaminants reflective of a highly complex mixture, ${ }^{5}$ we believe that these estimates reflected reasonable uncertainties caused by factors such as different tire formulations, TWP aging, environmental leaching rates, and contaminant attenuation after leaching. While we anticipate further increasing the accuracy of these HRMS estimates upon further method optimization, we believe the current estimates are credible, particularly with respect to relative comparisons, and also are consistent with peer reviewed literature with respect to absolute values. ${ }^{4,6}$ 


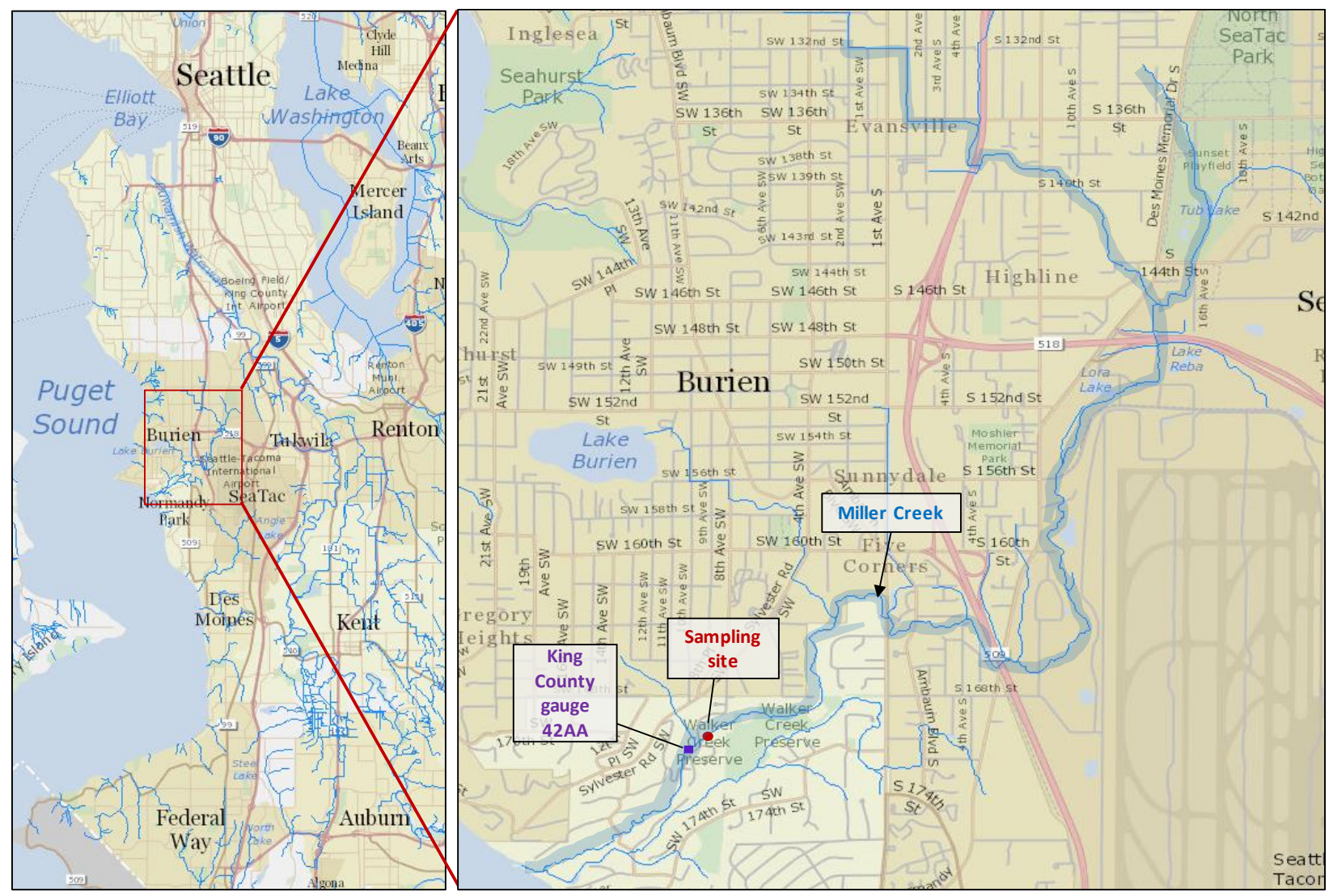

Figure S1. At left, Miller Creek watershed within the larger Seattle-Tacoma area. At right, Miller Creek watershed, with Miller Creek highlighted in blue and locations of the sampling site and King County gauge 42AA marked. Map from King County iMap. ${ }^{\mathbf{1 2}}$ 

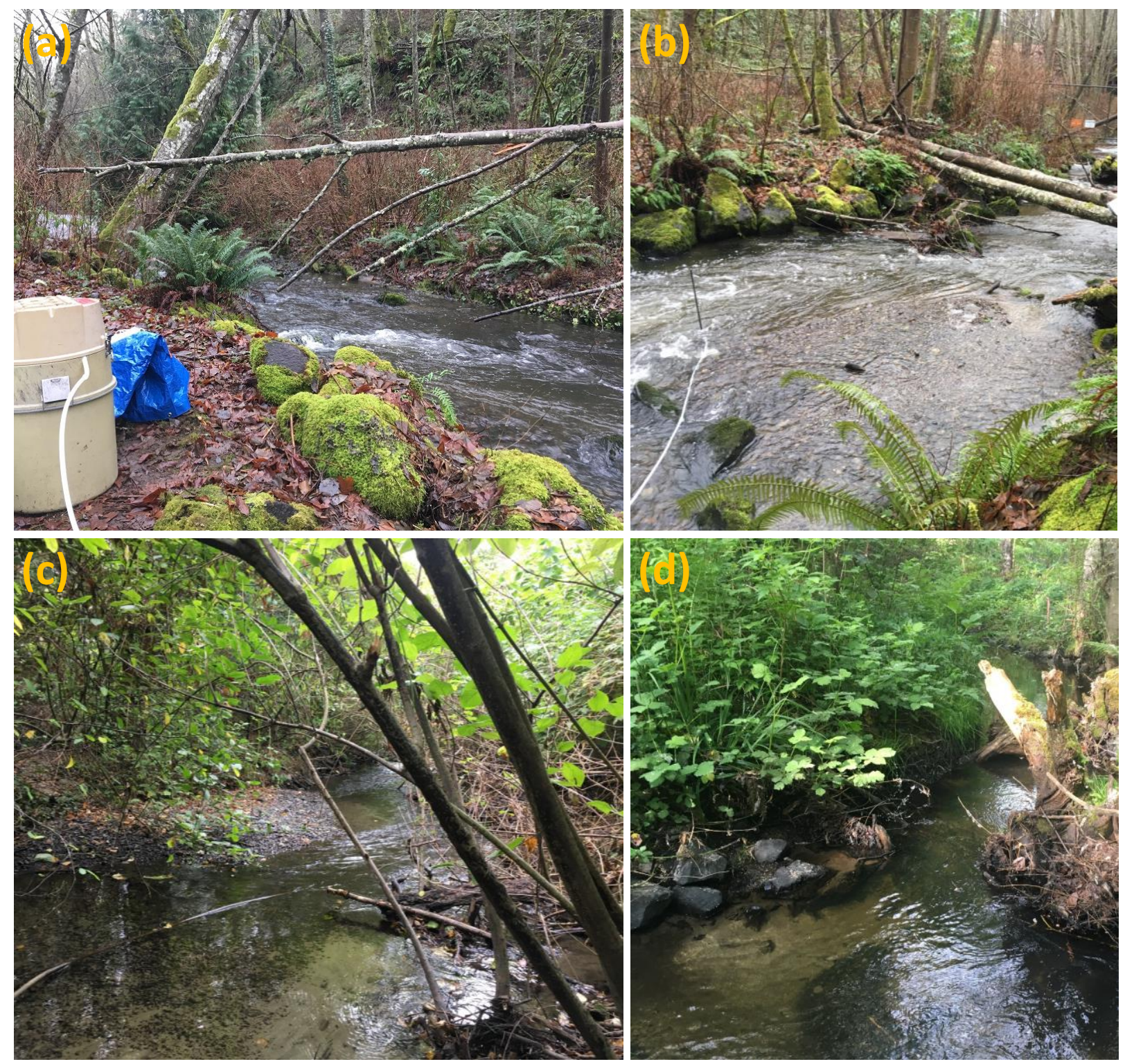

Figure S2. Miller Creek (a-b) at the sampling site on January 9, 2018, (c) $\sim 1.2 \mathrm{~km}$ downstream of the sampling site on September 25, 2017, and (d) $2.5 \mathrm{~km}$ upstream of the sampling site on May 8, 2018, showing the relatively well-restored appearance of this urban system. All photos were taken during baseflow conditions. Although photos were taken at different times, all are representative of the system appearance. 


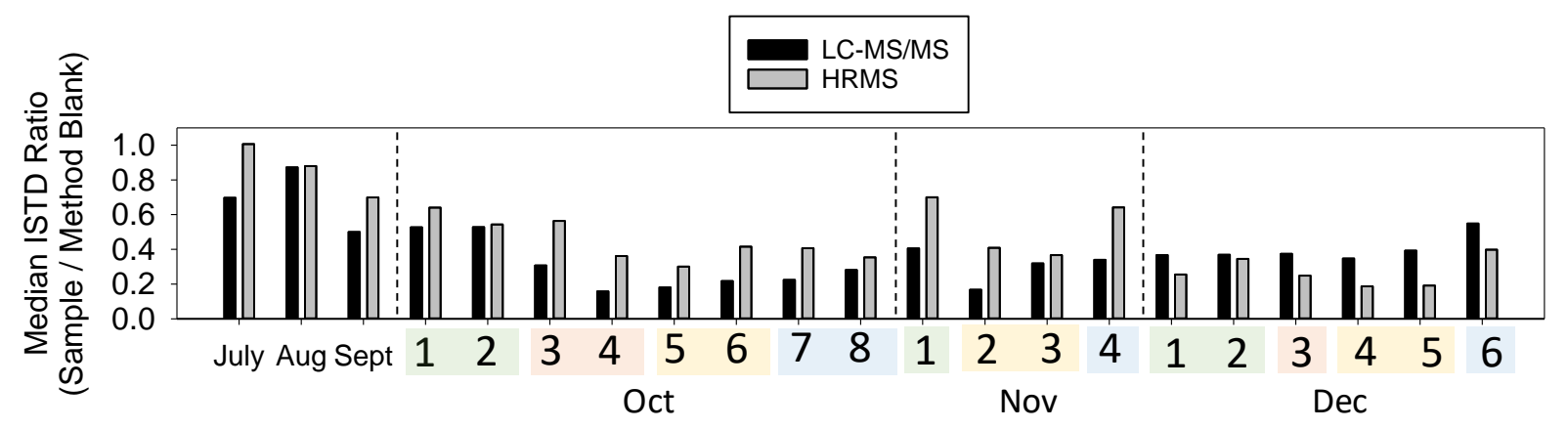

Figure S3. Median ratio of internal standard peak areas (sample / method blank) in LC-MS/MS $(\mathrm{n}=17)$ and HRMS $(\mathrm{n}=9)$ data. The ISTD ratios provide a gauge of relative matrix effects, and indicated increasing matrix effects with increasing stormflow across each storm hydrograph. Increasing matrix effects during the storm are attributed to increased contributions of suspended solids from both particulate matter transported in urban runoff and to erosion of creek banks by elevated, turbulent flows (a section of the creek bank upstream of the sampling site are relatively steep clay soils). Coloration denotes storm hydrograph segments based on rain accumulation and flow relative to peak discharge: pre-storm (green; $<5 \%$ total rain accumulation, $<0.5 \%$ total runoff volume); first flush (pink; 5-50\% rain accumulation, $0.5-10 \%$ runoff volume); storm peak (yellow; 50-100\% rain accumulation, 10-80\% runoff volume); and post-storm (blue; >80\% runoff volume). 


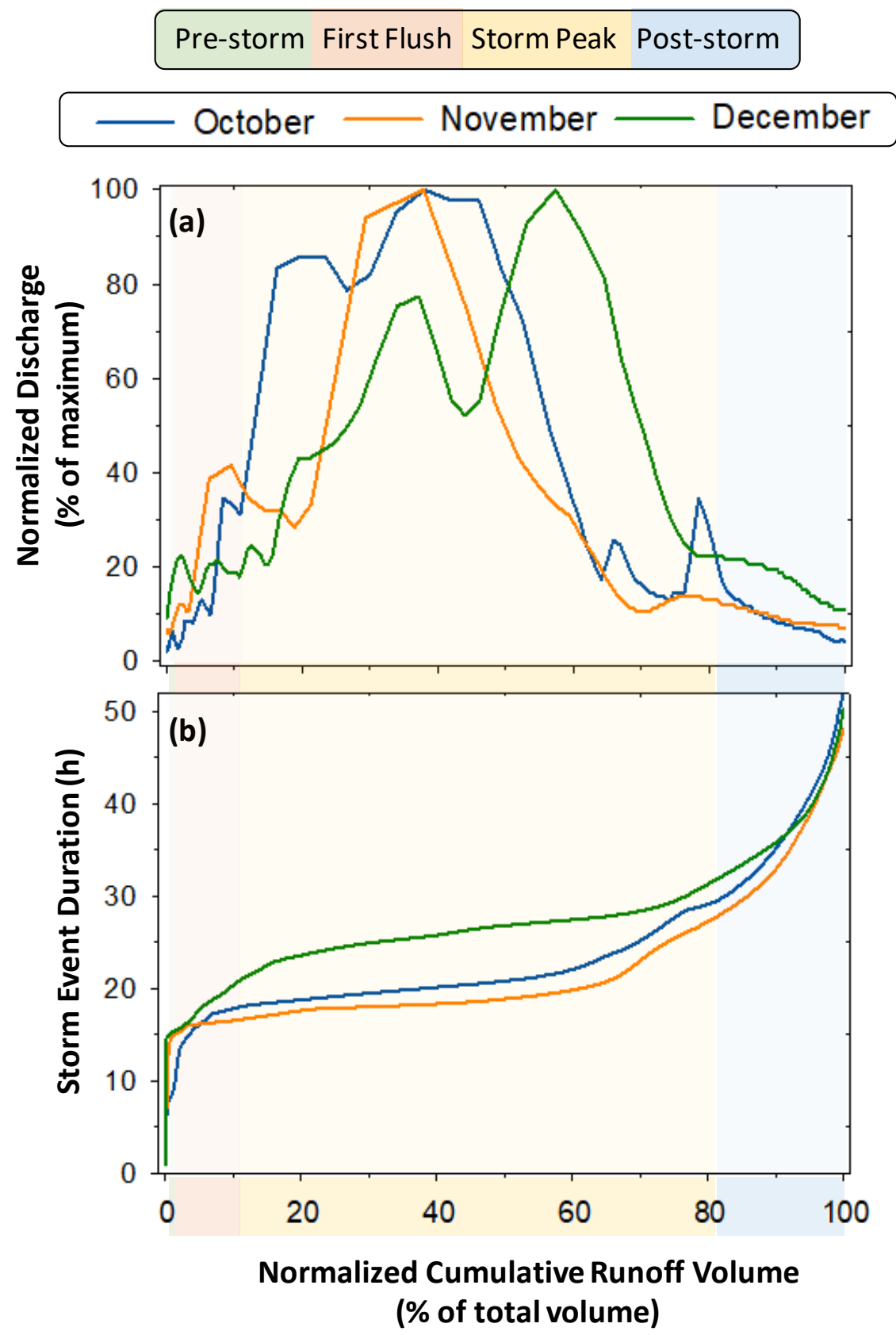

Figure S4. Hydrograph characteristics in Miller Creek during the three sampled storm events in October, November, and December 2018 as (a) normalized discharge (as a \% of maximum discharge) and (b) storm event duration (h) plotted against normalized cumulative runoff volume (as a $\%$ of total runoff volume). The later peak discharge during the December storm is evidenced by both the later peak discharge in (a) and the later (at $\sim 25 \mathrm{~h}$ vs. $\sim 18 \mathrm{~h}$ ) rapid increase in cumulative runoff volume in (b). Background coloration denotes hydrograph segments. 


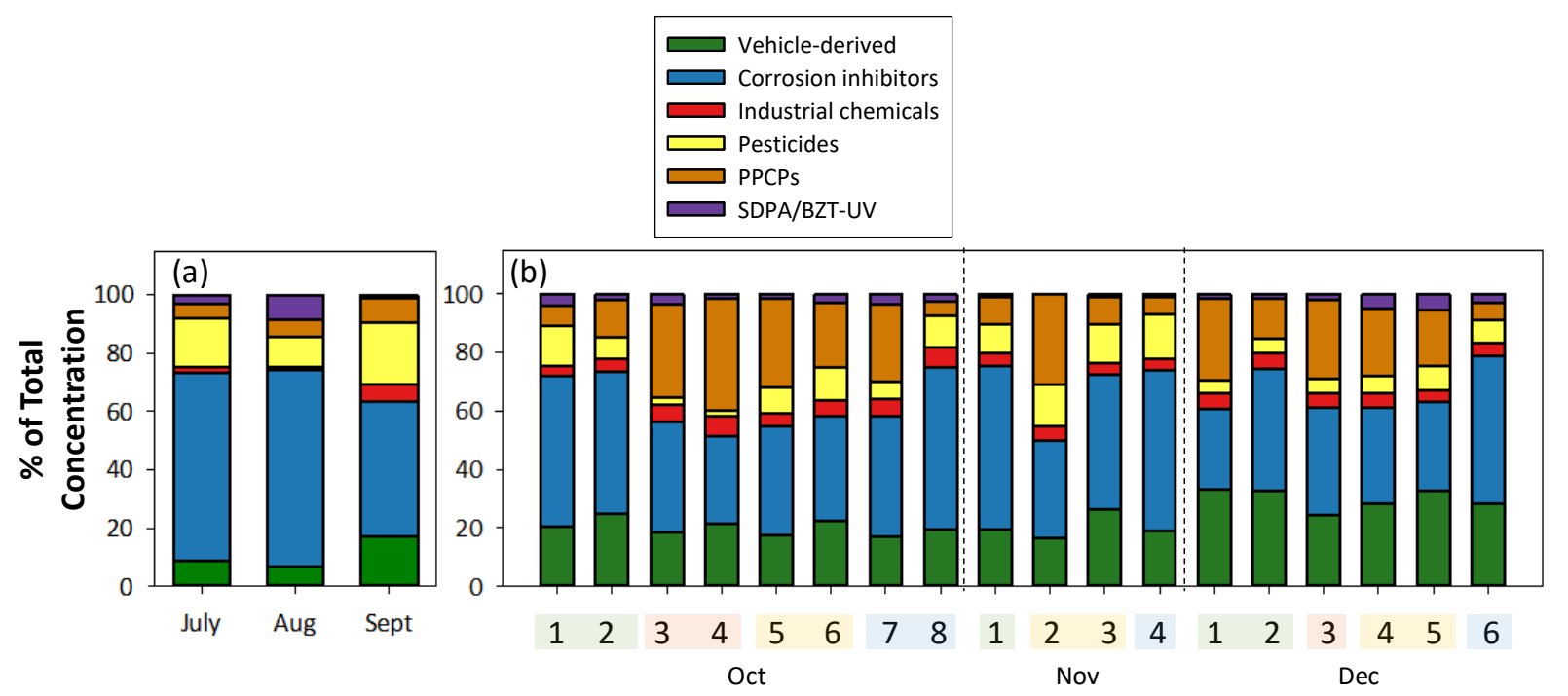

Figure S5. Proportion of total concentration contributed by each contaminant class during (a) baseflow and (b) storm events. Coloration of sample numbers denotes storm hydrograph segments based on rain accumulation and flow relative to peak discharge: pre-storm (green; $<5 \%$ total rain accumulation, $<0.5 \%$ total runoff volume); first flush (pink; 5-50\% rain accumulation, 0.5-10\% runoff volume); storm peak (yellow; 50-100\% rain accumulation, $10-80 \%$ runoff volume); and post-storm (blue; $>80 \%$ runoff volume). 


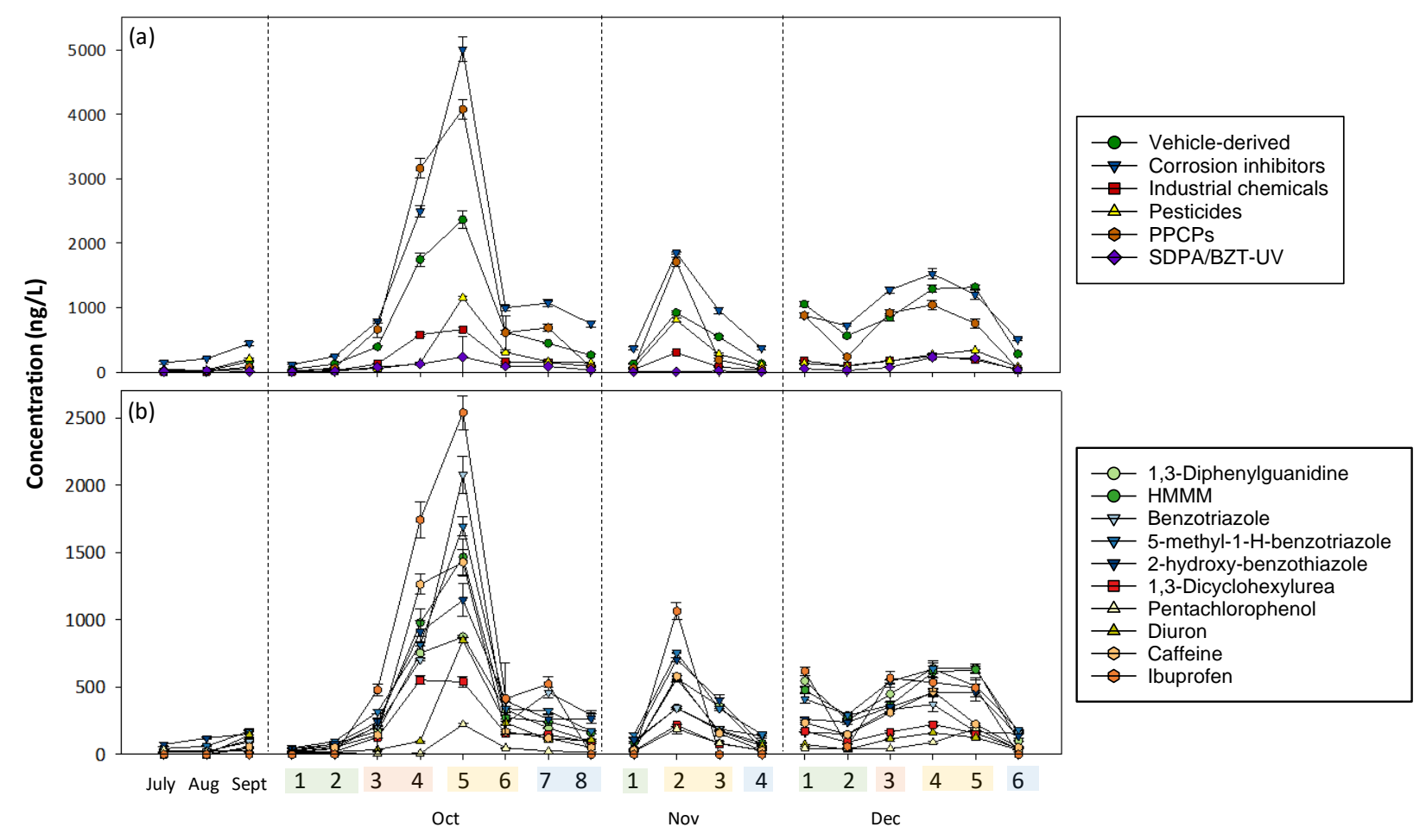

Figure S6. An alternative visual presentation of the data from Figure 3: (a) Concentrations of quantified analytes summed by contaminant class during baseflow and storm events. (b) Concentrations of select individual contaminants during baseflow and storm events, with the color corresponding to the contaminant class in panel (a). Contaminants shown in panel (b) accounted for $>80 \%$ of the total concentration in every sample. Coloration of sample numbers denotes storm hydrograph segments based on rain accumulation and flow relative to peak discharge: pre-storm (green; $<5 \%$ total rain accumulation, $<0.5 \%$ total runoff volume); first flush (pink; 5-50\% rain accumulation, $0.5-10 \%$ runoff volume); storm peak (yellow; $50-100 \%$ rain accumulation, $10-80 \%$ runoff volume); and post-storm (blue; >80\% runoff volume). 


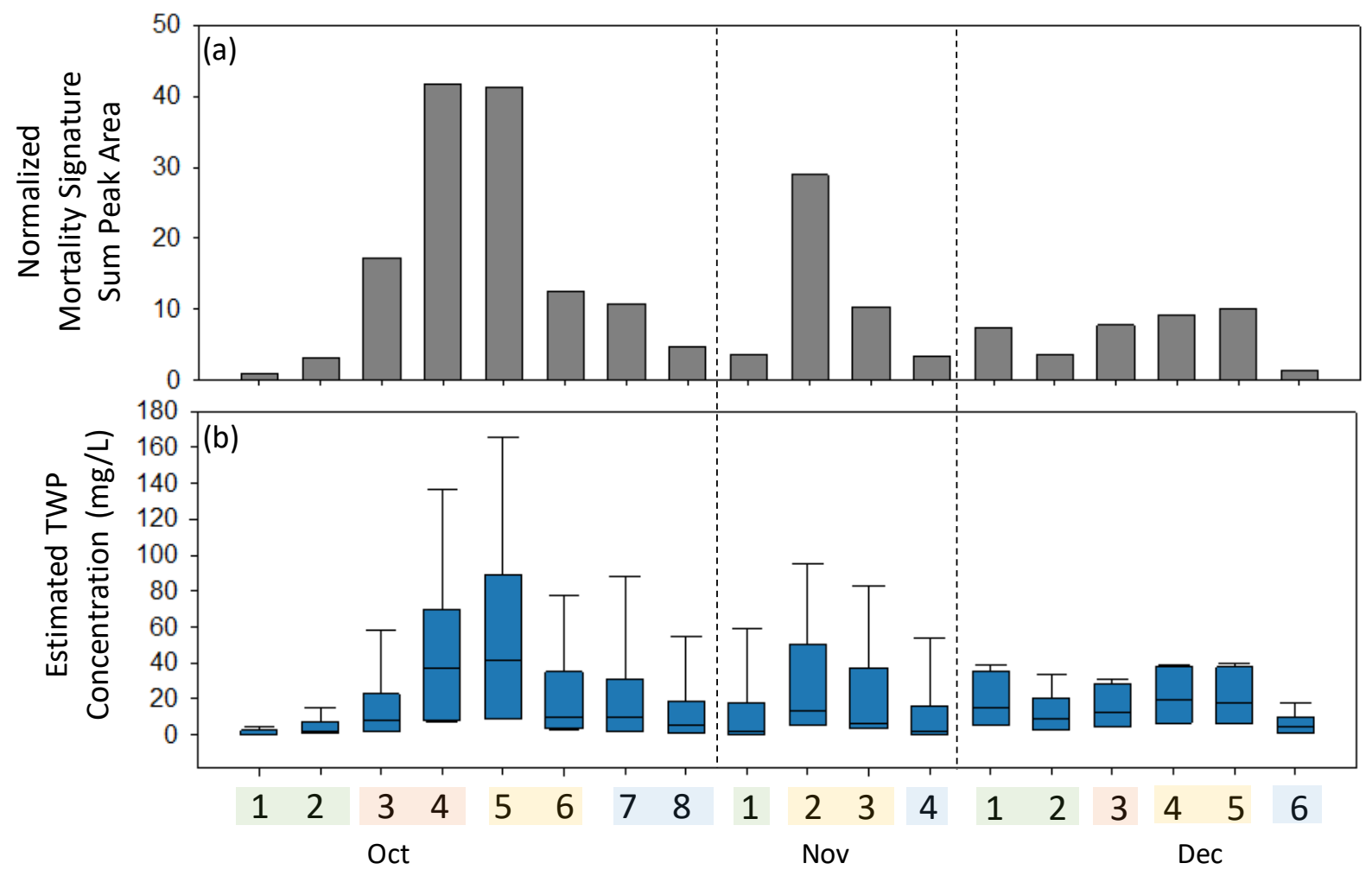

Figure S7. Surrogate metrics of water quality dynamics specific to coho salmon URMS, including the normalized URMS chemical signature and estimated TWP concentrations (data from Table 3). The normalized URMS signature represents the total peak area of all detected signature compounds, normalized to the minimum value observed across all storm event samples. The TWP concentrations ( $n=6$ estimates) reflect the relative quantity of TWPs needed to explain observed abundances of a mixture of dissolved TWP-derived contaminants, rather than direct estimates of particle concentration. The different TWP estimates were derived from a combination of HRMS data and quantified concentrations of TWP-derived contaminants. 


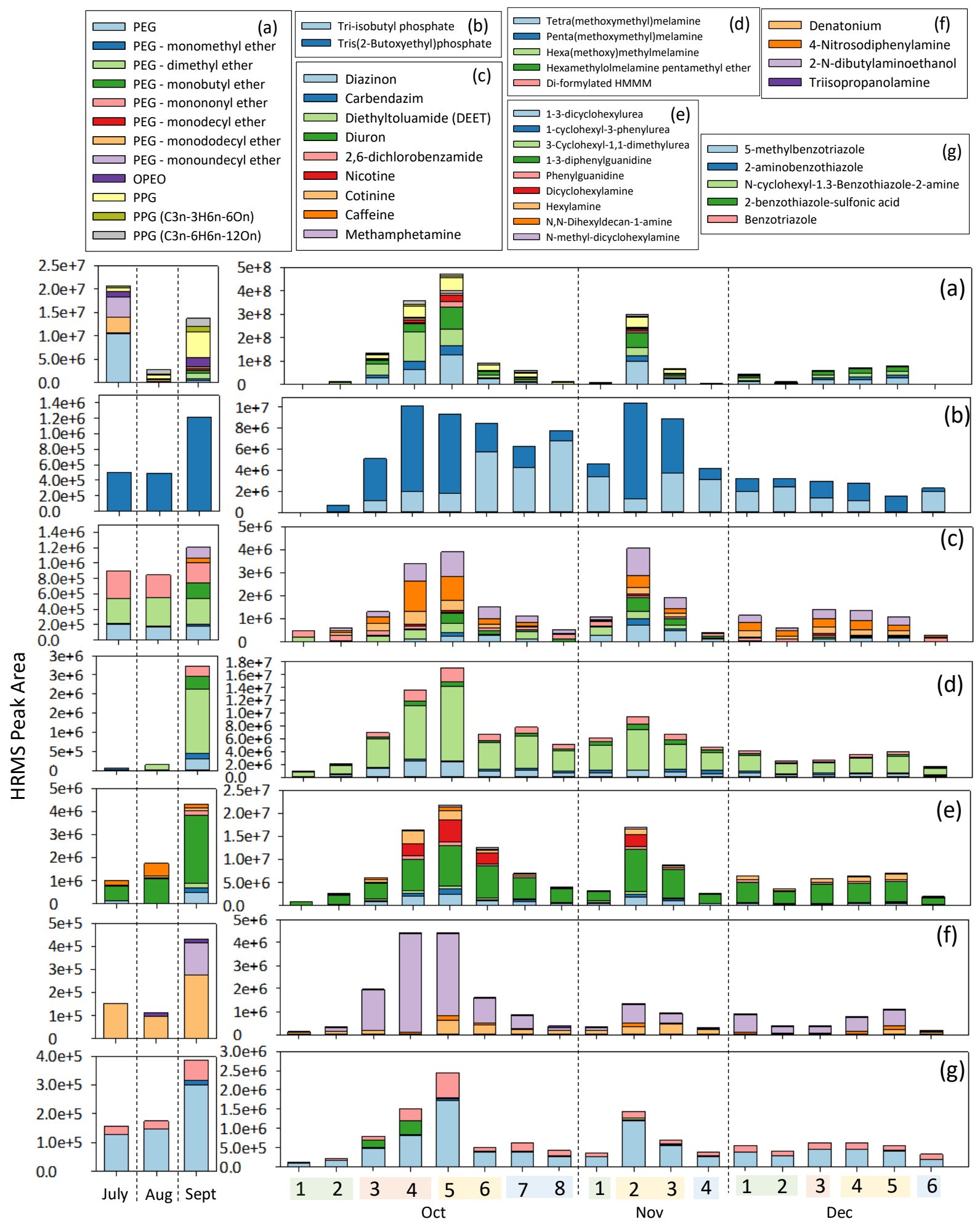

Figure S8. Peak area of identified HRMS compounds, including (a) long-chain surfactants, (b) organophosphates, (c) pesticides/pharmaceuticals, (d) (methoxymethyl)melamines, (e) vehiclederived compounds, (f) industrial compounds, and (g) corrosion inhibitors. 


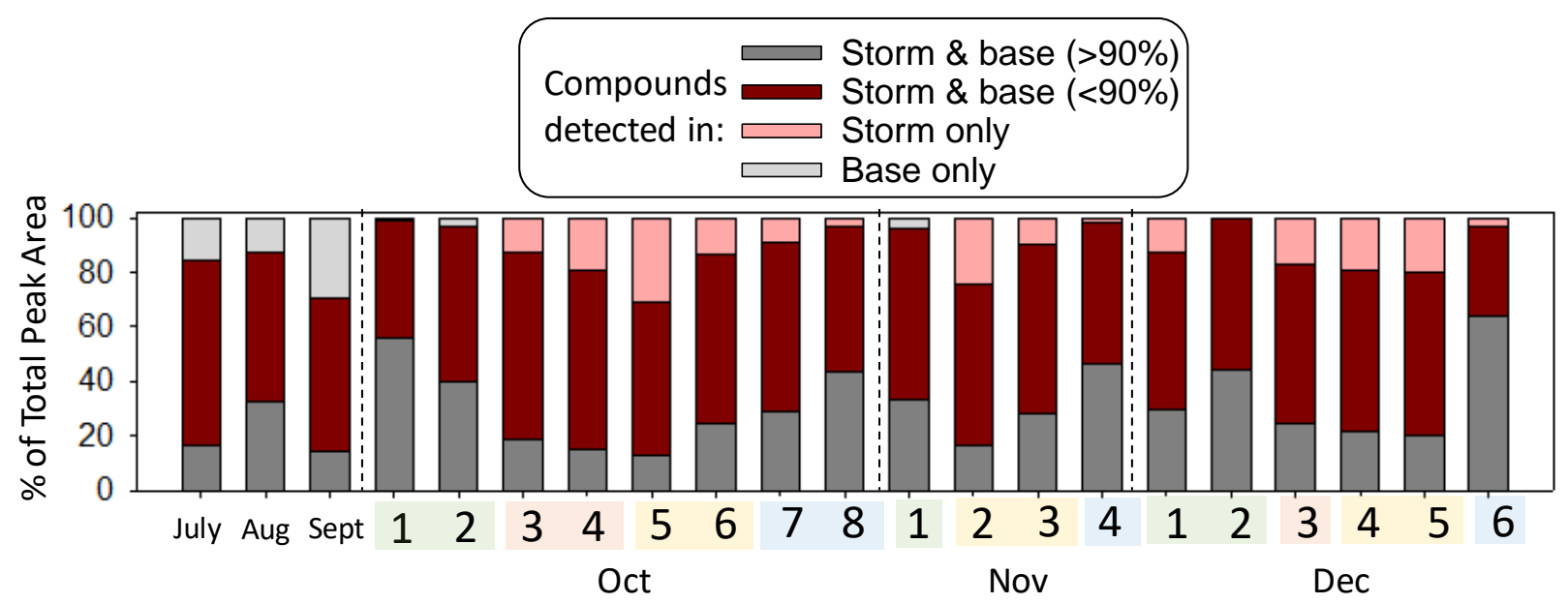

Figure S9. Proportion of total HRMS peak area in each sample attributed to compounds detected in baseflow/pre-storm conditions only, in stormflow conditions only, in multiple flow conditions ( $\geq 90 \%$ of all samples), or in multiple flow conditions ( $<90 \%$ of samples). Sample numbers and coloration on the $\mathrm{x}$-axis correspond to samples and hydrograph segments in Table 1 / Figure 1 (green $=$ pre-storm, pink $=$ first flush, yellow $=$ storm peak, and blue $=$ post-storm $)$. 

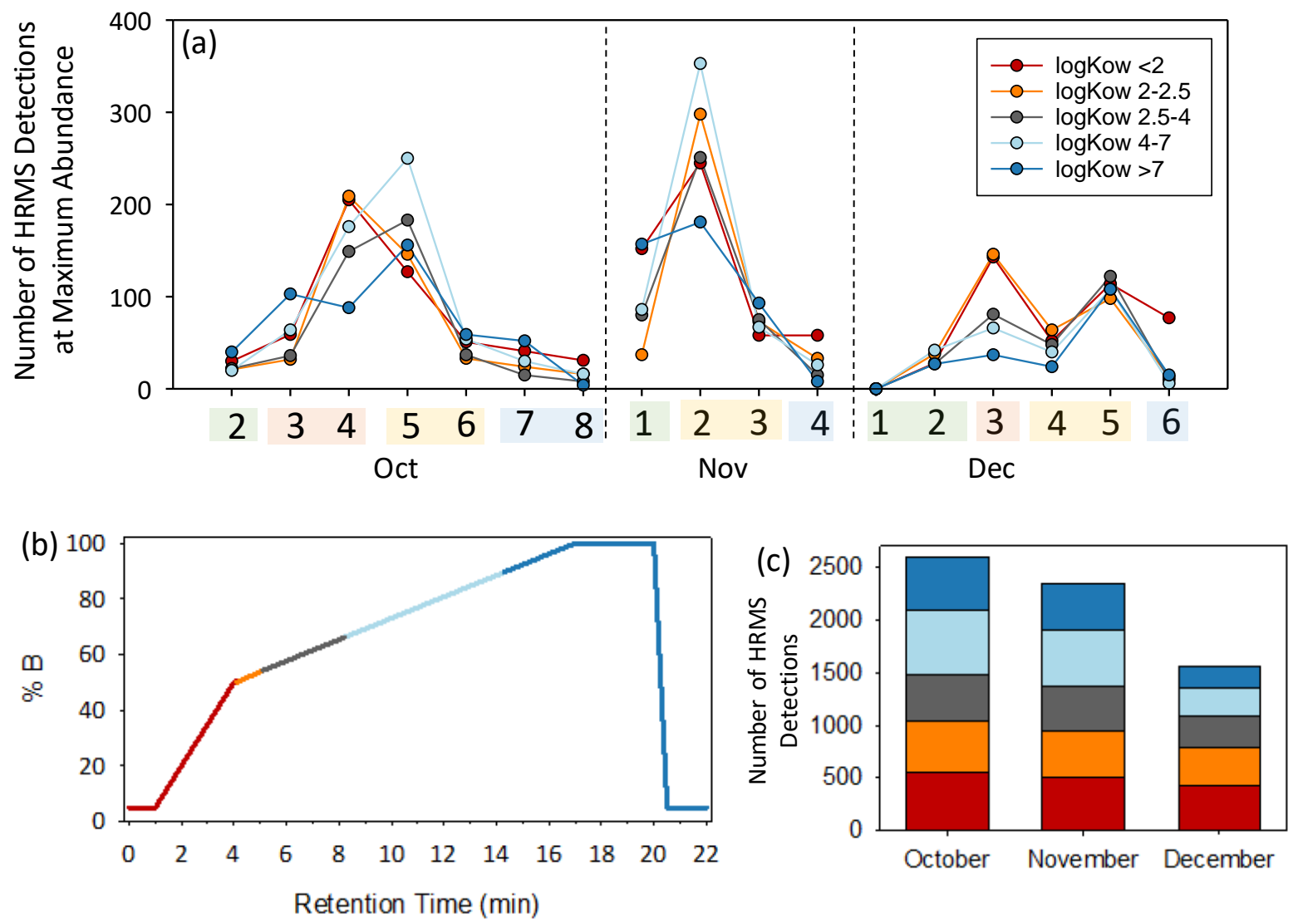

Figure S10. (a) Number of HRMS detections at maximum abundance in each storm sample (relative to other samples within each storm event) for October, November, and December 2018 storm events, shown by estimated polarity (as $\log K_{o w}$, using a previously established relationship between LC retention time and $\left.\log K_{o w}\right) ; ;^{13}$ (b) the relationship between the selected $\log K_{o w}$ ranges and LC retention time; and (c) the number of HRMS detections in each $\log K_{o w}$ segment for each storm event. To avoid skewing the trends, $\log K_{\text {ow }}$ ranges were selected in part to each contain a relatively equal number of detections. While evaluation of trends during the November storm was limited by the sampling resolution, a larger quantity of more-polar (lower $\log K_{o w}$ ) detections reached maximum abundance earlier in both October and December storm events, while a larger quantity of less-polar (higher $\log K_{\text {ow }}$ ) detections reached maximum abundance later in the storm events. These data indicated that molecular characteristics impacted transport outcomes, providing evidence that "watershed chromatography" occurred. We recommend further evaluation for confirmation of these trends. 


\section{References}

(1) Lu, Z.; De Silva, A. O.; Zhou, W.; Tetreault, G. R.; de Solla, S. R.; Fair, P. A.; Houde, M.; Bossart, G.; Muir, D. C. G. Substituted Diphenylamine Antioxidants and Benzotriazole UV Stabilizers in Blood Plasma of Fish, Turtles, Birds and Dolphins from North America. Sci. Total Environ. 2019, 647, 182-190. https://doi.org/10.1016/j.scitotenv.2018.07.405.

(2) Williams, A. J.; Grulke, C. M.; Edwards, J.; McEachran, A. D.; Mansouri, K.; Baker, N. C.; Patlewicz, G.; Shah, I.; Wambaugh, J. F.; Judson, R. S.; Richard, A. M. The CompTox Chemistry Dashboard: A Community Data Resource for Environmental Chemistry. $J$. Cheminformatics 2017, 9 (1). https://doi.org/10.1186/s13321-017-0247-6.

(3) Unice, K. M.; Bare, J. L.; Kreider, M. L.; Panko, J. M. Experimental Methodology for Assessing the Environmental Fate of Organic Chemicals in Polymer Matrices Using Column Leaching Studies and OECD 308 Water/Sediment Systems: Application to Tire and Road Wear Particles. Sci. Total Environ. 2015, 533, 476-487. https://doi.org/10.1016/j.scitotenv.2015.06.053.

(4) Unice, K. M.; Kreider, M. L.; Panko, J. M. Comparison of Tire and Road Wear Particle Concentrations in Sediment for Watersheds in France, Japan, and the United States by Quantitative Pyrolysis GC/MS Analysis. Environ. Sci. Technol. 2013, 130710100101002. https://doi.org/10.1021/es400871j.

(5) Peter, K. T.; Wu, C.; Tian, Z.; Kolodziej, E. P. Application of Nontarget High Resolution Mass Spectrometry Data to Quantitative Source Apportionment. Environ. Sci. Technol. 2019, 53 (21), 12257-12268. https://doi.org/10.1021/acs.est.9b04481.

(6) Wik, A.; Dave, G. Occurrence and Effects of Tire Wear Particles in the Environment - A Critical Review and an Initial Risk Assessment. Environ. Pollut. 2009, 157 (1), 1-11. https://doi.org/10.1016/j.envpol.2008.09.028.

(7) Reddy, C. M.; Quinn, J. G. Environmental Chemistry of Benzothiazoles Derived from Rubber. Environ. Sci. Technol. 1997, 31 (10), 2847-2853. https://doi.org/10.1021/es970078o.

(8) Kumata, H.; Yamada, J.; Masuda, K.; Takada, H.; Sato, Y.; Sakurai, T.; Fujiwara, K. Benzothiazolamines as Tire-Derived Molecular Markers: Sorptive Behavior in Street Runoff and Application to Source Apportioning. Environ. Sci. Technol. 2002, 36 (4), 702 708. https://doi.org/10.1021/es0155229.

(9) Kumata, H.; Sanada, Y.; Takada, H.; Ueno, T. Historical Trends of N-Cyclohexyl-2Benzothiazolamine, 2-(4-Morpholinyl)Benzothiazole, and Other Anthropogenic Contaminants in the Urban Reservoir Sediment Core. Environ. Sci. Technol. 2000, 34 (2), 246-253. https://doi.org/10.1021/es990738k.

(10) Ni, H.-G.; Lu, F.-H.; Luo, X.-L.; Tian, H.-Y.; Zeng, E. Y. Occurrence, Phase Distribution, and Mass Loadings of Benzothiazoles in Riverine Runoff of the Pearl River Delta, China. Environ. Sci. Technol. 2008, 42 (6), 1892-1897. https://doi.org/10.1021/es071871c.

(11) Wagner, S.; Hüffer, T.; Klöckner, P.; Wehrhahn, M.; Hofmann, T.; Reemtsma, T. Tire Wear Particles in the Aquatic Environment - a Review on Generation, Analysis, Occurrence, Fate and Effects. Water Res. 2018, 139, 83-100. https://doi.org/10.1016/j.watres.2018.03.051.

(12) King County. King County iMap https://www.kingcounty.gov/services/gis/Maps/imap.aspx.

(13) Du, B.; Lofton, J. M.; Peter, K. T.; Gipe, A. D.; James, C. A.; McIntyre, J. K.; Scholz, N. L.; Baker, J. E.; Kolodziej, E. P. Development of Suspect and Non-Target Screening 
Methods for Detection of Organic Contaminants in Highway Runoff and Fish Tissue with High-Resolution Time-of-Flight Mass Spectrometry. Env. Sci Process. Impacts 2017, 19, 1185. https://doi.org/10.1039/C7EM00243B. 